\title{
Massive Spinning Relativistic Particle: Revisited under BRST and Supervariable Approaches
}

\author{
A. Tripathi $\mathbb{D}^{1}{ }^{1}$ B. Chauhan $\mathbb{D}^{1},{ }^{1}$ A. K. Rao, ${ }^{1}$ and R. P. Malik $\mathbb{D}^{1,2}$ \\ ${ }^{1}$ Physics Department, Institute of Science, Banaras Hindu University, Varanasi 221 005, India \\ ${ }^{2}$ DST Centre for Interdisciplinary Mathematical Sciences, Institute of Science, Banaras Hindu University, Varanasi 221 005, India
}

Correspondence should be addressed to R. P. Malik; rpmalik1995@gmail.com

Received 1 July 2020; Accepted 29 July 2020; Published 17 August 2020

Academic Editor: Sunny Vagnozzi

Copyright (c) 2020 A. Tripathi et al. This is an open access article distributed under the Creative Commons Attribution License, which permits unrestricted use, distribution, and reproduction in any medium, provided the original work is properly cited. The publication of this article was funded by $\mathrm{SCOAP}^{3}$.

\begin{abstract}
We discuss the continuous and infinitesimal gauge, supergauge, reparameterization, nilpotent Becchi-Rouet-Stora-Tyutin (BRST), and anti-BRST symmetries and derive corresponding nilpotent charges for the one $(0+1)$-dimensional (1D) massive model of a spinning relativistic particle. We exploit the theoretical potential and power of the BRST and supervariable approaches to derive the (anti-)BRST symmetries and coupled (but equivalent) Lagrangians for this system. In particular, we capture the off-shell nilpotency and absolute anticommutativity of the conserved (anti-)BRST charges within the framework of the newly proposed (anti-)chiral supervariable approach (ACSA) to BRST formalism where only the (anti-)chiral supervariables (and their suitable super expansions) are taken into account along the Grassmannian direction(s). One of the novel observations of our present investigation is the derivation of the Curci-Ferrari- (CF-) type restriction by the requirement of the absolute anticommutativity of the (anti-)BRST charges in the ordinary space. We obtain the same restriction within the framework of ACSA to BRST formalism by (i) the symmetry invariance of the coupled Lagrangians and (ii) the proof of the absolute anticommutativity of the conserved and nilpotent (anti-)BRST charges. The observation of the anticommutativity property of the (anti-)BRST charges is a novel result in view of the fact that we have taken into account only the (anti-)chiral super expansions.
\end{abstract}

\section{Introduction}

The basic concepts behind the local gauge theories are at the heart of a precise theoretical description of three out of four fundamental interactions of nature. Becchi-Rouet-StoraTyutin (BRST) formalism [1-4] is one of the most intuitive and beautiful approaches to quantize the local gauge theories where the unitarity and quantum gauge (i.e., (anti-)BRST) invariance are respected together at any arbitrary order of perturbative computations for a given physical process that is permitted by the local (i.e., interacting) gauge theory at the quantum level. A couple of decisive features of the BRST formalism are the nilpotency of the (anti-)BRST symmetries as well as the existence of the absolute anticommutativity property between the BRST and anti-BRST symmetry transformations for a given local classical gauge transformation. The hallmark of the quantum (anti-)BRST symmetries is the existence of the (anti-)BRST invariant Curci-Ferrari-
(CF-) type restriction(s) $[5,6]$ that ensure the absolute anticommutativity property of the (anti-)BRST symmetry transformations and the existence of the coupled (but equivalent) Lagrangian densities for the quantum gauge theories. The Abelian 1-form gauge theory is an exception where the CFtype restriction is trivial and the Lagrangian density is unique (but that is a limiting case of the non-Abelian 1-form gauge theory where the CF condition [7] exists).

The usual superfield approach (USFA) to BRST formalism [8-15] sheds light on the geometrical origin for the offshell nilpotency and absolute anticommutativity of the (anti-)BRST symmetry transformations where the horizontality condition (HC) plays an important and decisive role [10-12]. These approaches, however, lead to the derivation of the (anti-)BRST symmetries for the gauge field and associated (anti-)ghost fields only [10-12]. The above USFA does not shed any light on the (anti-)BRST symmetries, associated with the matter fields, in an interacting gauge theory. In our 
earlier works (see, e.g., [16-19]), we have systematically and consistently generalized the USFA where in addition to the $\mathrm{HC}$, we exploit the potential and power of the gaugeinvariant restrictions (GIRs) to obtain the (anti-)BRST symmetry transformations for the matter, (anti-)ghost, and gauge fields of an interacting gauge theory together. There is no conflict between the HC and GIRs as they complement and supplement each other in a beautiful fashion. This approach has been christened as the augmented version of superfield approach (AVSA) to BRST formalism. The USFA with $\mathrm{HC}$, developed in [10-12], is mathematically very elegant and, in one stroke, it leads to the derivation of proper (anti-)BRST symmetry transformations for the gauge and associated (anti-)ghost fields along with the derivation of the (anti-)BRST invariant CF-condition. The AVSA is a minor extension of [10-12] where $\mathrm{HC}$ and gauge invariant restrictions are exploited together (cf. Section 8 ).

In the recent set of papers [20-24], we have developed a simpler version of the AVSA where only the (anti-)chiral supervariables/superfields and their appropriate super expansions have been taken into consideration. This superfield approach to BRST formalism has been christened as the (anti-)chiral superfield/supervariable approach (ACSA). It may be mentioned here that, in all the earlier superfield approaches [8-19], the full super expansions of the superfields/supervariables, along all the Grassmannian directions of the $(\mathrm{D}, 2)$-dimensional supermanifold, have been taken into account for the consideration of a D-dimensional local gauge-invariant theory (defined on the flat Minkowskian space). One of the decisive features of the ACSA to BRST formalism is its dependence on the quantum gauge (i.e., (anti-)BRST) invariant restrictions on the supervariables/superfields which lead to the derivation of appropriate (anti)BRST symmetry transformations for all the fields/variables of the theory together with the deduction of the (anti-)BRST invariant CF-type restriction(s). The upshot of the results from ACSA is the observation that the conserved and nilpotent (anti-)BRST charges turn out to be absolutely anticommuting in nature despite the fact that only the (anti-)chiral super expansions of the supervariables/superfields are taken into account (within the framework of ACSA to BRST formalism).

The purpose of our present investigation is to apply the ACSA to BRST formalism to the $1 \mathrm{D}$ system of a massive spinning relativistic particle and derive the proper (anti-) BRST symmetry transformations for this system so that it can be discussed and described within the framework of BRST formalism. Our present 1D reparameterization invariant system is important in its own right as it provides a prototype model for the (super)gauge-invariant theory as well as an example for a toy model of the supergravity theory. Needless to say, its generalization leads to the theory of superstrings, too. If the existence of the continuous symmetries is the guiding principle for the definition of a beautiful theory in physics, the $1 \mathrm{D}$ model of a massive spinning particle represents one such example which encompasses in its folds a host of beautiful continuous symmetries (cf. Sections 2 and 3 ). In our present investigation, we lay a whole lot of emphasis on the off-shell nilpotent and absolutely anticommuting
(anti-)BRST symmetry transformations of our 1D system and derive the corresponding conserved Noether charges. It is worthwhile to mention, at this stage, that, physically, the property of off-shell nilpotency of the (anti-)BRST symmetries and corresponding charges imply their fermionic nature and the absolute anticommutativity property encodes the linear independence of the above nilpotent symmetries and charges.

Against the backdrop of the above discussions, in our present endeavor, we have shown the existence of the three classical level symmetries which are the gauge, supergauge, and reparameterization transformations (cf. Equations (2) and (4)) under which the first-order Lagrangian $\left(L_{f}\right)$ for the 1D system of a massive spinning relativistic particle remains invariant. We have further established that the reparameterization symmetry transformations contain (i) the gauge symmetry transformations (cf. Equation (2)) provided all the fermionic variables are set equal to zero (i.e., $\psi_{\mu}=\psi_{5}=\chi=0$ ), and (ii) the combination of gauge and supergauge symmetry transformations (cf. Equation (7)) under specific conditions where the appropriate equations of motion and identifications of the transformation parameters are taken into account (cf. Equations (6), (9), and (10). We have elevated the classical (super)gauge symmetry transformations (7) to the quantum level within the framework of BRST formalism and derived the (anti-)BRST symmetries that are respected by the coupled (but equivalent) Lagrangians $L_{b}$ and $L_{\bar{b}}$ (cf. Equations (17) and (18)). We have demonstrated that both the Lagrangians are equivalent because both of them respect both the BRST and anti-BRST symmetry transformations at the quantum level provided the whole theory is considered on the submanifold of the quantum Hilbert space of variables where the CF-type restriction is satisfied (cf. Equations (20), (21), (22), and (23)). We have further shown the existence of the (anti-)BRST invariant CF-type restriction at the level of the proof of absolute anticommutativity of the (anti-)BRST conserved charges in the ordinary space (cf. Equations (35), (36), (37), and (38)).

In our present endeavor, we have captured all the above key features within the framework of ACSA to BRST formalism where only the (anti-)chiral supervariables and their corresponding super expansion(s) along the Grassmannian direction(s) of the $(1,1)$-dimensional (anti-)chiral super submanifolds of the general $(1,2)$-dimensional supermanifold have been taken into consideration in a consistent and systematic fashion. One of the novel observations is the proof of the absolute anticommutativity property of the conserved and nilpotent (anti-)BRST charges within the ambit of ACSA to BRST formalism where only the (anti-)chiral super expansion(s) of the (anti-)chiral supervariables have been taken into account. Moreover, we note that the above proof also distinguishes between the chiral and antichiral $(1,1)$-dimensional super submanifolds within the framework of ACSA to BRST formalism (cf. Appendix D).

Our present investigation is essential and interesting on the following counts. First and foremost, our 1D system of the massive spinning relativistic particle is more general than its massless counterpart which has been discussed in our earlier work [25]. Second, our present system is a toy model of a 
supersymmetric gauge theory whose generalization to $4 \mathrm{D}$ provides a model for the supergravity theory with a cosmological constant term. Hence, this toy model is interesting and important in its own right. Third, our present model is also a generalization of the scalar relativistic particle where the fermionic as well as bosonic (anti-)ghost variables appear within the framework of BRST formalism. Fourth, we have been curious to find out the contribution of the mass term (and its associated variable) in the determination of the gauge-fixing and Faddeev-Popov ghost terms within the framework of BRST formalism (cf. Equation (16)). Fifth, we have found out the CF-type restriction for the 1D massless spinning particle in our earlier work by exploiting the beauty of the supersymmetrization of horizontality condition [25]. Thus, we are now curious to find out its existence by proving the absolute anticommutativity of the conserved (anti-)BRST charges. Furthermore, we are interested in capturing its existence within the framework of ACSA to BRST formalism. We have accomplished all these goals in our present endeavor. Finally, a thorough study of our 1D system of a massive spinning relativistic has been a challenge for us as we have already studied a scalar relativistic particle and a massless spinning relativistic particle from various angels in our earlier works [25-32].

The theoretical material of our present endeavor is organized as follows. In Section 2, we discuss the gauge, supergauge, and reparameterization symmetries of the Lagrangian that describes the $1 \mathrm{D}$ massive spinning relativistic particle. Our Section 3 deals with the (anti-)BRST symmetries corresponding to the combined gauge and supergauge symmetries where the fermionic as well as the bosonic (anti-)ghost variables appear in the BRST analysis. The subject matter of Section 4 concerns itself with the derivation of the BRST symmetries within the framework of ACSA to BRST formalism where the quantum gauge (i.e., BRST) invariant restrictions on the antichiral supervariables play a crucial role. Our Section 5 is devoted to the derivation of anti-BRST symmetries by exploiting the anti-BRST invariant restrictions on the chiral supervariables within the purview of ACSA to BRST formalism. In Section 6, we prove the existence of the CF-type restriction by capturing the symmetry invariance of the Lagrangians within the ambit of ACSA. We capture the off-shell nilpotency and absolute anticommutativity of the conserved (anti-)BRST charges by applying the key techniques of ACSA to BRST formalism in Section 7. Finally, in Section 8, we make some concluding remarks and point out a few future directions for further investigations.

In our Appendices A, B, and C, we collect a few of the explicit computations which supplement as well as complement some of the crucial and key statements that have been made and emphasized in the main body of our present endeavor. Our Appendix D is devoted to the discussion of an alternative proof of the absolute anticommutativity of the (anti-)BRST charges and the existence of the CF-type restriction (i) in the ordinary space and (ii) in the superspace by exploiting the theoretical tricks and techniques of ACSA.

Convention and notations: the free (i.e., $\dot{p}_{\mu}=0$ ) massive spinning relativistic particle is embedded in a D-dimensional flat Minkowskian spacetime manifold that is characterized by a metric tensor $\eta_{\mu \nu}=\operatorname{diag}(+1,-1,-1 \cdots)$ where the Greek indices $\mu, v, \lambda \cdots=0,1,2 \cdots(D-1)$. We adopt the convention of the left-derivative w.r.t. the fermionic variables $\left(\chi, \psi_{\mu}\right.$, $\left.\psi_{5}, \gamma, c, \bar{c}\right)$. We denote the (anti-)BRST fermionic $\left(s_{(a) b}^{2}=0\right)$ symmetry transformations by the symbol $s_{(a) b}$ which anticommutes (i.e., $\chi s_{(a) b}=-s_{(a) b} \chi, s_{(a) b} \psi_{\mu}=-\psi_{\mu} s_{(a) b}$, etc.) with all the fermionic variables $\left(\chi, \psi_{\mu}, \psi_{5}, \gamma, c, \bar{c}\right)$ and commutes (i.e., $x_{\mu} s_{(a) b}=s_{(a) b} x_{\mu}, \quad p_{\mu} s_{(a) b}=s_{(a) b} p_{\mu}, \quad e s_{(a) b}=s_{(a) b} e, \quad s_{(a) b} \bar{b}=$ $\bar{b} s_{(a) b}$, etc.) with all the bosonic variables of our theory. We also denote the (anti-)BRST charges by the symbol $Q_{(a) b}$.

\section{Preliminaries: Some Continuous and Infinitesimal Symmetries in Lagrangian Formulation}

In this section, we discuss some infinitesimal and continuous symmetries and demonstrate their equivalence under some specific conditions where the usefulness of some appropriate equations of motion as well as identifications of a few transformation parameters has been exploited. We begin with the following three equivalent Lagrangians which describe the 1D system of a massive spinning relativistic particle (see, e.g., [33])

$$
\begin{aligned}
L_{0}= & m \sqrt{\left(\dot{x}^{\mu}+i \chi \psi^{\mu}\right)\left(\dot{x}_{\mu}+i \chi \psi_{\mu}\right)}-i m \chi \psi_{5}, \\
L_{f}= & p_{\mu} \dot{x}^{\mu}+\frac{i}{2}\left(\psi_{\mu} \dot{\psi}^{\mu}-\psi_{5} \dot{\psi}_{5}\right)-\frac{e}{2}\left(p^{2}-m^{2}\right) \\
& +i \chi\left(p_{\mu} \psi^{\mu}-m \psi_{5}\right), \\
L_{s}= & \frac{1}{2 e}\left(\dot{x}_{\mu}+i \chi \psi_{\mu}\right)\left(\dot{x}^{\mu}+i \chi \psi^{\mu}\right)+\frac{e}{2} m^{2} \\
& +\frac{i}{2}\left(\psi_{\mu} \dot{\psi}^{\mu}-\psi_{5} \dot{\psi}_{5}\right)-i m \chi \psi_{5},
\end{aligned}
$$

where $L_{0}$ is the Lagrangian with a square root, $L_{f}$ is the firstorder Lagrangian, and $L_{s}$ is the second-order Lagrangian. Our one $(0+1)$-dimensional (1D) system is embedded in a flat Minkowskian D-dimensional target space where $\left(x_{\mu}, p^{\mu}\right)$ are the canonically conjugate bosonic coordinates and momenta (with $\mu=0,1,2 \cdots D-1$ ). The trajectory of the particle is parameterized by an evolution parameter $\tau$, and generalized velocities $\left(\dot{x}_{\mu}=d x_{\mu} / d \tau, \dot{\psi}_{\mu}=d \psi_{\mu} / d \tau\right)$ are defined w.r.t. it. We have fermionic $\left(\psi_{\mu} \psi_{\nu}+\psi_{\nu} \psi_{\mu}=0, \quad \chi \psi_{\mu}+\psi_{\mu} \chi\right.$ $=0, \quad \psi_{\mu} \psi_{5}+\psi_{5} \psi_{\mu}=0, \chi \psi_{5}+\psi_{5} \chi=0, \chi^{2}=0$, etc.) variables in our theory which commute $\left(\psi_{\mu} e-e \psi_{\mu}=0, \psi_{\mu} x_{\nu}-x_{\nu} \psi_{\mu}\right.$ $=0, \psi_{\mu} p_{v}-p_{\nu} \psi_{\mu}=0, \dot{x}_{v} \psi_{\mu}-\psi_{\mu} \dot{x}_{v}=0$, etc.) with all the bosonic variables $\left(x_{\mu}, p_{\mu}, e\right)$ of our theory. It should be noted that $\psi_{\mu}$ is the superpartner of $x_{\mu}$ and $\psi_{5}$ variable has been invoked in the theory to incorporate a mass term $m$ so that the mass-shell condition $\left(p^{2}-m^{2}=0\right)$ for the free particle could be satisfied. We would like to point out that, in Ref. [33], the emphasis is laid on the first-order Lagrangians and their usefulness. Hence, the first-order Lagrangian $\left(L_{f}\right)$ is the 
only Lagrangian that is mentioned in [33] and there is absence of $L_{0}$ as well as $L_{s}$.

The Lagrangian $L_{0}$ has a square root, and its massless limit is not defined. On the other hand, the second-order Lagrangian $\left(L_{s}\right)$ is endowed with a variable (i.e., einbein) which is located in the denominator. Thus, the Lagrangians $L_{0}$ and $L_{s}$ have their own limitations. We shall focus on the first-order Lagrangian $\left(L_{f}\right)$ for our discussions where variables $e(\tau)$ and $\chi(\tau)$ are not purely Lagrange multiplier variables but their transformations are such that they behave like the "gauge" and "supergauge" variables (cf. Equation (2)). Our 1D system is a model of supersymmetric gauge theory, and its generalization to $4 \mathrm{D}$ theory provides a model for the supergravity theory where $\psi_{\mu}$ corresponds to the RaritaSchwinger field and $e(\tau)$ becomes the vierbein field. The mass $m$, in the supergravity theory, represents the cosmological constant term. In a nutshell, our present 1D model of a massive spinning relativistic particle is important and interesting in its own right because its generalization also becomes a model of the superstring theory (see, e.g., [34, 35]).

The Lagrangian $L_{f}$ respects the following gauge $\left(\delta_{g}\right)$ and supergauge $\left(\delta_{s g}\right)$ symmetry transformations, namely,

$$
\begin{aligned}
\delta_{g} x_{\mu} & =\xi p_{\mu}, \\
\delta_{g} p_{\mu} & =0, \\
\delta_{g} e & =\dot{\xi}, \\
\delta_{g} \psi_{\mu} & =0, \\
\delta_{g} \psi_{5} & =0, \\
\delta_{g} \chi & =0, \\
\delta_{s g} x_{\mu} & =\kappa \psi_{\mu}, \\
\delta_{s g} \psi_{\mu} & =i \kappa p_{\mu}, \\
\delta_{s g} p_{\mu} & =0, \\
\delta_{s g} \psi_{5} & =i \kappa m, \\
\delta_{s g} \chi & =i \dot{\kappa}, \\
\delta_{s g} e & =2 \kappa \chi,
\end{aligned}
$$

where $\xi(\tau)$ and $\kappa(\tau)$ are the infinitesimal gauge and supergauge symmetry transformation parameters, respectively. It is straightforward to note that $\xi(\tau)$ is a bosonic and $\kappa(\tau)$ is a fermionic (i.e., $\kappa^{2}=0$ ) transformation parameter. Furthermore, the transformation $\delta_{s g}$ is a supersymmetric transformation because it transforms a bosonic variable to a fermionic variable and vice versa. The transformations in Equation (2) are symmetry transformations because the first-order Lagrangian $L_{f}$ transforms to the following total derivatives:

$$
\begin{aligned}
\delta_{g} L_{f} & =\frac{d}{d \tau}\left[\frac{\xi}{2}\left(p^{2}+m^{2}\right)\right], \\
\delta_{s g} L_{f} & =\frac{d}{d \tau}\left[\frac{\kappa}{2}\left(p_{\mu} \psi^{\mu}+m \psi_{5}\right)\right] .
\end{aligned}
$$

As a consequence, it is clear that the action integral $S=$ $\int_{-\infty}^{+\infty} d \tau L_{f}$, under the transformations $\delta_{g}$ and $\delta_{s g}$, would be equal to zero (i.e., $\delta_{p} S=0, p=g, s g$ ) due to the fact that all the physical variables vanish off at $\tau= \pm \infty$. There is a reparameterization symmetry, too, in our theory due to the basic infinitesimal transformation $\tau \rightarrow \tau^{\prime}=\tau-\varepsilon(\tau)$ where $\varepsilon(\tau)$ is an infinitesimal transformation parameter. In fact, the physical variables of our $1 \mathrm{D}$ system transform under the infinitesimal reparameterization transformation $\left(\delta_{r}\right)$ as

$$
\begin{aligned}
\delta_{r} x_{\mu} & =\varepsilon \dot{x}_{\mu}, \\
\delta_{r} p_{\mu} & =\varepsilon \dot{p}_{\mu}, \\
\delta_{r} \psi_{\mu} & =\varepsilon \dot{\psi}_{\mu}, \\
\delta_{r} \psi_{5} & =\varepsilon \dot{\psi}_{5}, \\
\delta_{r} e & =\frac{d}{d \tau}(\varepsilon e), \\
\delta_{r} \chi & =\frac{d}{d \tau}(\varepsilon \chi) .
\end{aligned}
$$

The above transformations are symmetry transformations for the action integral $S=\int_{-\infty}^{+\infty} d \tau L_{f}$ because of the following transformation property of $L_{f}$, namely,

$$
\delta_{r} L_{f}=\frac{d}{d \tau}\left[\varepsilon L_{f}\right] \Longrightarrow \delta_{r} S=0 .
$$

It is evident that $\delta_{r} S=0$ due to the fact that $\epsilon(\tau)$ and $L_{f}$ vanish off at $\tau= \pm \infty$.

The reparameterization symmetry transformation $\left(\delta_{r}\right)$ and gauge symmetry transformation $\left(\delta_{g}\right)$ are equivalent under the following limits:

$$
\begin{aligned}
\xi & =\epsilon e, \\
\dot{x}_{\mu} & =e p_{\mu}, \\
\dot{p}_{\mu} & =0,
\end{aligned}
$$

provided we set all the fermionic variables $\left(\chi, \psi_{5}, \psi_{\mu}\right)$ of our theory equal to zero. In the above, we have used equations of motion: $\dot{x}_{\mu}=e p_{\mu}$ and $\dot{p}_{\mu}=0$, and we have identified the gauge symmetry transformation parameter $\xi(\tau)$ with the combination of the reparameterization transformation parameter $\epsilon(\tau)$ and the einbein variable $e(\tau)$. In an exactly similar fashion, we note that $\left(\delta_{r}\right)$ and $\left(\delta_{g}+\delta_{s g}\right)$ are also equivalent. In this context, first of all, we note that there are two primary constraints (i.e., $\Pi_{e} \approx 0, \Pi_{\chi} \approx 0$ ) and two secondary constraints (i.e., $p^{2}-m^{2} \approx 0, p_{\mu} \psi^{\mu}-m \psi_{5} \approx 0$ ) on our theory where $\Pi_{e}$ and $\Pi_{\chi}$ are the canonical conjugate momenta w.r.t. the Lagrange multiplier variables $e$ and $\chi$, respectively. The above four constraints of our theory are first-class in the terminology of Dirac's prescription for the classification scheme of constraints because they (anti)commute among themselves [36, 37]. These constraints generate 
the combined (super)gauge symmetry transformations $\delta=\delta_{g}+\delta_{s g}$ for the physical variables of our theory as (see, e.g., [27])

$$
\begin{aligned}
\delta x_{\mu} & =\xi p_{\mu}+\kappa \psi_{\mu} \\
\delta p_{\mu} & =0 \\
\delta \psi_{\mu} & =i \kappa p_{\mu} \\
\delta e & =\dot{\xi}+2 \kappa \chi \\
\delta \chi & =i \dot{\kappa}, \\
\delta \psi_{5} & =i \kappa m
\end{aligned}
$$

under which the first-order Lagrangian $L_{f}$ transforms to a total "time" derivative as

$$
\delta L_{f}=\frac{d}{d \tau}\left[\frac{\xi}{2}\left(p^{2}+m^{2}\right)+\frac{\kappa}{2}\left(p_{\mu} \psi^{\mu}+m \psi_{5}\right)\right] .
$$

As a consequence of the above observation, it is evident that $\delta S=0$ where $S=\int_{-\infty}^{+\infty} d \tau L_{f}$ is the action integral. If we use the following equations of motion:

$$
\begin{aligned}
& \dot{p}_{\mu}=0, \\
& \dot{x}_{\mu}=e p_{\mu}-i \chi \psi_{\mu}, \\
& \dot{\psi}_{\mu}=\chi p_{\mu}, \\
& \dot{\psi}_{5}=m \chi,
\end{aligned}
$$

and identify the transformation parameters as

$$
\begin{aligned}
& \xi=e \varepsilon, \\
& \kappa=-i \varepsilon \chi
\end{aligned}
$$

we find that the reparameterization symmetry transformation (4) (emerging due to the basic transformation: $\tau \rightarrow \tau^{\prime}=\tau-\varepsilon$ $(\tau))$ and the combined gauge and supergauge symmetry transformations (i.e., $\delta=\delta_{g}+\delta_{s g}$ ), quoted in Equation (7), are equivalent to each other. It is worthwhile to note that, under the identifications (10), the transformation $\delta e=\dot{\xi}+2 \kappa \chi$ becomes $\delta e=(d / d \tau)(\varepsilon e)$ as we note that $2 \kappa \chi=-2 i e \chi^{2}=0$.

We end this section with the following remarks. First of all, we note that the canonical Hamiltonians, derived from $L_{0}$ and $L_{f}$ (as well as $L_{s}$ ), are

$$
\begin{aligned}
H_{c}^{(0)} & =-i \chi\left(p_{\mu} \psi^{\mu}-m \psi_{5}\right), \\
H_{c} & =\frac{e}{2}\left(p^{2}-m^{2}\right)-i \chi\left(p_{\mu} \psi^{\mu}-m \psi_{5}\right),
\end{aligned}
$$

where $H_{c}^{(0)}$ is the canonical Hamiltonian corresponding to the Lagrangian $L_{0}$. It is straightforward to note that the primary constraints $\Pi_{e} \approx 0, \Pi_{\chi} \approx 0$ lead to the derivation of the secondary constraints $\left(p^{2}-m^{2}\right) \approx 0,\left(p_{\mu} \psi^{\mu}-m \psi_{5}\right) \approx 0$ from the Hamiltonians (11) as well as from all the three equivalent Lagrangians (1) (cf. Appendix A). Second, we have explicitly demonstrated that the (super)gauge symmetry transformations and reparameterization symmetry transformations are equivalent under specific conditions (cf. Equations (9) and (10)). Finally, the system under consideration is very interesting and important because it is endowed with many symmetries and it provides a prototype example for the supersymmetric gauge theory, superstrings, and a model for the supergravity theory.

\section{3. (Anti-)BRST Symmetries: Lagrangian Formulation}

Our present section is divided into two parts. In Subsection 3.1, we show the existence of the CF-type restriction by the requirement of absolute anticommutativity of the (anti-)BRST symmetries and (anti-)BRST invariance of the coupled (but equivalent) Lagrangians $L_{b}$ and $L_{\bar{b}}$. In Subsection 3.2, we establish the existence of the same by requiring the absolute anticommutativity of the conserved and nilpotent (anti-) BRST charges.

3.1. (Anti-)BRST Invariance and CF-Type Restriction. Corresponding to the combined classical (super)gauge symmetry transformations (cf. Equation (7)), we can write down the quantum (anti-)BRST symmetry transformations $s_{(a) b}$ where the classical gauge symmetry parameter $\xi(\tau)$ would be replaced by the fermionic $\left(c^{2}=\bar{c}^{2}=0, c \bar{c}+\bar{c} c=0\right.$, etc.) (anti-)ghost variables $(\bar{c}) c$ and the classical supergauge symmetry transformations parameter $\kappa(\tau)$ would be replaced by a pair of bosonic $\left(\beta^{2}=\bar{\beta} \neq 0\right)$ (anti-)ghost variables $(\bar{\beta}) \beta$. These off-shell nilpotent $\left[\left(s_{(a) b}\right)^{2}=0\right]$, infinitesimal and continuous (anti-)BRST symmetry transformations $\left(s_{(a) b}\right)$, in their full blaze of glory for our 1D system of the massive spinning relativistic particle, are (see, e.g., [25])

$$
\begin{aligned}
s_{a b} x_{\mu} & =\bar{c} p_{\mu}+\bar{\beta} \psi_{\mu}, \\
e & =\dot{\bar{c}}+2 \bar{\beta} \chi, \\
s_{a b} \psi_{\mu} & =i \bar{\beta} p_{\mu}, \\
s_{a b} \bar{c} & =-i \bar{\beta}^{2}, \\
s_{a b} c & =i \bar{b}, \\
s_{a b} \bar{\beta} & =0, \\
s_{a b} \beta & =-i \gamma, \\
s_{a b} p_{\mu} & =0, \\
s_{a b} \gamma & =0, \\
s_{a b} \bar{b} & =0, \\
s_{a b} \chi & =i \dot{\bar{\beta}}, \\
s_{a b} b & =2 i \bar{\beta} \gamma,
\end{aligned}
$$




$$
\begin{aligned}
s_{a b} \psi_{5} & =i \bar{\beta} m, \\
s_{b} x_{\mu} & =c p_{\mu}+\beta \psi_{\mu}, \\
s_{b} e & =\dot{c}+2 \beta \chi, \\
s_{b} \psi_{\mu} & =i \beta p_{\mu}, \\
s_{b} c & =-i \beta^{2}, \\
s_{b} \bar{c} & =i b, \\
s_{b} \beta & =0, \\
s_{b} \bar{\beta} & =i \gamma, \\
s_{b} p_{\mu} & =0, \\
s_{b} \gamma & =0, \\
s_{b} b & =0, \\
s_{b} \chi & =i \dot{\beta}, \\
s_{b} \bar{b} & =-2 i \beta \gamma, \\
s_{b} \psi_{5} & =i \beta m,
\end{aligned}
$$

where $b$ and $\bar{b}$ are the Nakanishi-Lautrup-type auxiliary variables, fermionic $\left(\chi^{2}=0, c^{2}=\bar{c}^{2}=0, \gamma^{2}=0\right)$ variables $(\chi, c, \bar{c}$, $\gamma)$ are present in our theory, and the rest of the symbols have already been explained earlier. As far as the absolute anticommutativity $\left(s_{b} s_{a b}+s_{a b} s_{b}=0\right)$ property is concerned, it can be checked that

$$
\begin{aligned}
\left\{s_{b}, s_{a b}\right\} x_{\mu} & =i(b+\bar{b}+2 \beta \bar{\beta}) p_{\mu}, \\
\left\{s_{b}, s_{a b}\right\} e & =i \frac{d}{d \tau}(b+\bar{b}+2 \beta \bar{\beta}),
\end{aligned}
$$

are equal to zero only after imposing the CF-type restriction: $b+\bar{b}+2 \beta \bar{\beta}=0$ from outside. It is worthwhile to mention that this CF-type restriction is a physical restriction within the realm of BRST formalism because it is an (anti-)BRST invariant (i.e., $\left.s_{(a) b}[b+\bar{b}+2 \beta \bar{\beta}]=0\right)$ quantity. Except for the variables $\left(x_{\mu}, e\right)$, it is straightforward to check that the following is true for the other variables of our theory, namely,

$$
\begin{aligned}
\left\{s_{b}, s_{a b}\right\} \Phi & =0, \\
\Phi & =p_{\mu}, \psi_{\mu}, \psi_{5}, \chi, \beta, \bar{\beta}, c, \bar{c}, b, \bar{b}, \gamma,
\end{aligned}
$$

where $\Phi(\tau)$ is the generic variable of the (anti-)BRST invariant theory. Thus, it is crystal clear that the (anti-) BRST symmetry transformations in (12) and (13) are offshell nilpotent $\left[\left(s_{(a) b}\right)^{2}=0\right]$ and absolutely anticommuting $\left(s_{b} s_{a b}+s_{a b} s_{b}=0\right)$ in nature provided the whole theory is considered on a submanifold of space of quantum variables where the CF-type restriction: $b+\bar{b}+2 \beta \bar{\beta}=0$, is satisfied in the quantum Hilbert space (see, e.g., [25]).
The coupled (but equivalent) Lagrangians for our (anti-) BRST invariant system of the $1 \mathrm{D}$ massive spinning relativistic particle can be written as

$$
\begin{aligned}
& L_{b}=L_{f}+s_{b} s_{a b}\left[\frac{i e^{2}}{2}+c \bar{c}+\chi \psi_{5}\right], \\
& L_{\bar{b}}=L_{f}-s_{a b} s_{b}\left[\frac{i e^{2}}{2}+c \bar{c}+\chi \psi_{5}\right],
\end{aligned}
$$

where $L_{f}$ is the first-order Lagrangian that has been quoted in Equation (1). The above Lagrangians for our 1D system of a massive spinning relativistic particle can be written, in their full glory incorporating the gauge-fixing and FaddeevPopov ghost terms, as

$$
\begin{aligned}
L_{b}= & L_{f}+b^{2}+b(\dot{e}+2 \bar{\beta} \beta)-\dot{\bar{c}} \dot{c}+\bar{\beta}^{2} \beta^{2}+2 i \chi(\beta \dot{\bar{c}}-\bar{\beta} \dot{c}) \\
& -2 e(\bar{\beta} \dot{\beta}+\gamma \chi)+2 \gamma(\beta \bar{c}-\bar{\beta} c)+m(\bar{\beta} \dot{\beta}-\dot{\bar{\beta}} \beta+\gamma \chi)-\dot{\gamma} \psi_{5}, \\
L_{\bar{b}}= & L_{f}+\bar{b}^{2}-\bar{b}(\dot{e}-2 \bar{\beta} \beta)-\dot{i \bar{c} \dot{c}}+\bar{\beta}^{2} \beta^{2}+2 i \chi(\beta \dot{\bar{c}}-\bar{\beta} \dot{c}) \\
& +2 e(\dot{\bar{\beta}} \beta-\gamma \chi)+2 \gamma(\beta \bar{c}-\bar{\beta} c)+m(\bar{\beta} \dot{\beta}-\dot{\bar{\beta}} \beta+\gamma \chi)-\dot{\gamma} \psi_{5},
\end{aligned}
$$

where, as pointed out earlier, $b$ and $\bar{b}$ are the NakanishiLautrup-type auxiliary variables which lead to the derivation of EL-EOMs (from $L_{b}$ and $L_{\bar{b}}$ ) as

$$
\begin{aligned}
& 2 b+\dot{e}+2 \beta \bar{\beta}=0, \\
& 2 \bar{b}-\dot{e}+2 \beta \bar{\beta}=0 .
\end{aligned}
$$

It is elementary to note that the above relationships lead to the derivation of the CF-type restriction: $b+\bar{b}+2 \bar{\beta} \beta=0$, which is the hallmark of a quantum gauge theory discussed within the framework of BRST formalism $[5,6]$.

At this juncture, we are in the position to focus on the symmetry properties of the coupled Lagrangians $L_{b}$ and $L_{\bar{b}}$. In this context, we observe the following:

$$
s_{b} L_{b}=\frac{d}{d \tau}\left[\frac{c}{2}\left(p^{2}+m^{2}\right)+\frac{\beta}{2}\left(p_{\mu} \psi^{\mu}+m \psi_{5}\right)+b(\dot{c}+2 \beta \chi)\right] \text {, }
$$

$$
s_{a b} L_{\bar{b}}=\frac{d}{d \tau}\left[\frac{\bar{c}}{2}\left(p^{2}+m^{2}\right)+\frac{\bar{\beta}}{2}\left(p_{\mu} \psi^{\mu}+m \psi_{5}\right)-\bar{b}(\dot{\bar{c}}+2 \bar{\beta} \chi)\right] .
$$

It is clear from the above observations that the action integrals $S_{1}=\int_{-\infty}^{\infty} d \tau L_{b}$ and $S_{2}=\int_{-\infty}^{\infty} d \tau L_{\bar{b}}$ remain invariant (i.e., $s_{b} S_{1}=0, s_{a b} S_{2}=0$ ) under the quantum BRST and antiBRST symmetry transformations that have been listed in Equations (13) and (12). The coupled (but equivalent) Lagrangian respect both (i.e., BRST and anti-BRST) quantum symmetries provided the whole theory is considered on a 
submanifold of the quantum Hilbert space of variables where the CF-type restriction: $b+\bar{b}+2 \beta \bar{\beta}=0$, is satisfied. In other words, mathematically, we observe the following:

$$
\begin{aligned}
s_{b} L_{\bar{b}}= & \frac{d}{d \tau}\left[\frac{c}{2}\left(p^{2}+m^{2}\right)+\frac{\beta}{2}\left(p_{\mu} \psi^{\mu}+m \psi_{5}\right)-\bar{b}(\dot{c}+2 \beta \chi)+2 i e \beta \gamma\right] \\
& +(\dot{c}+2 \beta \chi)\left[\frac{d}{d \tau}(b+\bar{b}+2 \beta \bar{\beta})\right]-(2 i \beta \gamma)(b+\bar{b}+2 \beta \bar{\beta})
\end{aligned}
$$

$$
\begin{aligned}
s_{a b} L_{b}= & \frac{d}{d \tau}\left[\frac{\bar{c}}{2}\left(p^{2}+m^{2}\right)+\frac{\bar{\beta}}{2}\left(p_{\mu} \psi^{\mu}+m \psi_{5}\right)+b(\dot{\bar{c}}+2 \bar{\beta} \chi)+2 i e \bar{\beta} \gamma\right] \\
& -(\dot{\bar{c}}+2 \bar{\beta} \chi)\left[\frac{d}{d \tau}(b+\bar{b}+2 \beta \bar{\beta})\right]+(2 i \bar{\beta} \gamma)(b+\bar{b}+2 \beta \bar{\beta}) .
\end{aligned}
$$

A close look at the above transformations demonstrates that if we impose the (anti-)BRST invariant $\left[s_{(a) b}(b+\bar{b}+2 \beta\right.$ $\bar{\beta})=0$ ] quantum CF-type restriction $(b+\bar{b}+2 \beta \bar{\beta}=0)$ from outside, we obtain the following BRST symmetry transformation of the Lagrangian $L_{\bar{b}}$ and anti-BRST symmetry transformation of the Lagrangian $L_{b}$, namely,

$$
s_{b} L_{\bar{b}}=\frac{d}{d \tau}\left[\frac{c}{2}\left(p^{2}+m^{2}\right)+\frac{\beta}{2}\left(p_{\mu} \psi^{\mu}+m \psi_{5}\right)-\bar{b}(\dot{c}+2 \beta \chi)+2 i e \beta \gamma\right],
$$

$s_{a b} L_{b}=\frac{d}{d \tau}\left[\frac{\bar{c}}{2}\left(p^{2}+m^{2}\right)+\frac{\bar{\beta}}{2}\left(p_{\mu} \psi^{\mu}+m \psi_{5}\right)+b(\dot{\bar{c}}+2 \bar{\beta} \chi)+2 i e \bar{\beta} \gamma\right]$.

It is crystal clear now that the observations in Equations (20), (21), (22), (23), (24), and (25) imply, in a straightforward manner, that both the Lagrangians (i.e., $L_{b}$ and $L_{\bar{b}}$ ) respect both the quantum symmetries (i.e., BRST and antiBRST symmetry transformations) in the space of quantum variables where the CF-type restriction is satisfied.

We end this subsection with the following remarks. First and foremost, we observe that the presence of the term " $\chi \psi_{5}$ " in the square bracket of Equation (16) is due to the massive nature of the spinning relativistic particle. In the massless case, it disappears (see, e.g., Ref. [25]). Second, the hallmark of the quantum gauge theory (within the framework of the BRST formalism) is encoded in the existence of the CF-type restriction which we have demonstrated in Equations (14), (19), (22), and (23) where we have concentrated on the quantum (anti-)BRST symmetries which are respected by the coupled Lagrangians $L_{b}$ and $L_{\bar{b}}$. Finally, we note that the absolute anticommutativity property of the (anti-)BRST symmetries and equivalence of $L_{b}$ and $L_{\bar{b}}$ owe their origins to the CF-type restriction: $b+\bar{b}+2 \beta \bar{\beta}=0$.

3.2. (Anti-)BRST Charges and CF-Type Restriction. In this subsection, we demonstrate the existence of the (anti-)BRST invariant CF-type restriction (i.e., $b+\bar{b}+2 \beta \bar{\beta}=0$ ) by demanding the absolute anticommutativity of the conserved and nilpotent (anti-)BRST charges of our present theory. In this context, first of all, we note that, according to Noether's theorem, the invariances $s_{b} S_{1}=0, s_{a b} S_{2}=0$ of the action integrals $S_{1}=\int_{-\infty}^{+\infty} d \tau L_{b}$ and $S_{2}=\int_{-\infty}^{+\infty} d \tau L_{\bar{b}}$ under the (anti-)BRST symmetry transformations $\left(s_{(a) b}\right)$ (as quoted in Equations (20) and (21)) lead to the derivation of the Noether conserved (anti-)BRST charges $\left(Q_{(a) b}^{(1)}\right)$ as follows:

$$
\begin{aligned}
Q_{a b}^{(1)}= & \frac{\bar{c}}{2}\left(p^{2}-m^{2}\right)+\bar{\beta}\left(p_{\mu} \psi^{\mu}-m \psi_{5}\right)-\bar{b} \dot{\bar{c}}-2 \bar{b} \bar{\beta} \chi \\
& -i m \bar{\beta} \gamma-\bar{\beta}^{2} \dot{c}-2 \beta \bar{\beta}^{2} \chi, \\
Q_{b}^{(1)}= & \frac{c}{2}\left(p^{2}-m^{2}\right)+\beta\left(p_{\mu} \psi^{\mu}-m \psi_{5}\right)+b \dot{c}+2 b \beta \chi \\
& -i m \beta \gamma+\beta^{2} \dot{\bar{c}}+2 \bar{\beta} \beta^{2} \chi .
\end{aligned}
$$

The conservation law (i.e., $\dot{Q}_{b}^{(1)}=0, \dot{Q}_{a b}^{(1)}=0$ ) can be proven by using the EL-EOMs derived from the Lagrangians $L_{b}$ and $L_{\bar{b}}$ (cf. Appendix B). We have used the superscript (1) on the (anti-)BRST charges $\left(Q_{(a) b}^{(1)}\right)$ to denote that these charges have been directly derived by using the basic principle behind Noether's theorem. However, we have the option of expressing these charges in a different form by using the ELEOMs that are derived from $L_{b}$ and $L_{\bar{b}}$. At this stage, it can be noted that the Noether conserved charges $Q_{(a) b}^{(1)}$ are not off-shell nilpotent $\left(\left[Q_{(a) b}^{(1)}\right]^{2} \neq 0\right)$ of order two without any use of EL-EOMs. In other words, we note that the following is true, namely,

$$
\begin{gathered}
s_{b} Q_{b}^{(1)}=-i\left\{Q_{b}^{(1)}, Q_{b}^{(1)}\right\} \neq 0, \\
s_{a b} Q_{a b}^{(1)}=-i\left\{Q_{a b}^{(1)}, Q_{a b}^{(1)}\right\} \neq 0,
\end{gathered}
$$

unless we use the EL-EOMs from $L_{b}$ and $L_{\bar{b}}$. Thus, we lay emphasis on the fact that $Q_{(a) b}^{(1)}$ are only the on-shell nilpotent conserved charges (even though we have used the off-shell nilpotent (anti-)BRST symmetry transformations (12) and (13) in their derivation).

We have the freedom to use the EL-EOMs (derived from $L_{\bar{b}}$ and $\left.L_{b}\right)$ to recast the Noether conserved charges $Q_{(a) b}^{(1)}$ in a different form. For instance, the BRST charge $Q_{b}^{(1)}$ can be written in a different form by using the following EL-EOMs:

$$
\begin{aligned}
\dot{b} & =-\frac{1}{2}\left(p^{2}-m^{2}\right)-2(\bar{\beta} \dot{\beta}+\gamma \chi), \\
p_{\mu} \psi^{\mu}-m \psi_{5} & =2 i e \gamma-2(\beta \dot{\bar{c}}-\bar{\beta} \dot{c}),
\end{aligned}
$$

which are derived from $L_{b}$ w.r.t. the $e$ and $\chi$ variables. The ensuing expression for the conserved BRST charge (due to EL-EOMs (29)) is

$$
\begin{aligned}
Q_{b}^{(2)}= & b \dot{c}-\dot{b} c+2 i e \beta \gamma+2 \beta \bar{\beta} \dot{c}-2 c(\bar{\beta} \dot{\beta}+\gamma \chi)+2 b \beta \chi \\
& -2 i m \gamma \beta-\beta^{2} \dot{\bar{c}}+2 \chi \beta^{2} \bar{\beta}
\end{aligned}
$$


Here, the superscript (2) denotes that the expression for the BRST charge in Equation (30) has been derived from the Noether conserved BRST charge $Q_{b}^{(1)}$ by using the ELEOMs quoted in Equation (29). It is now straightforward to check that the following is true, namely,

$$
s_{b} Q_{b}^{(2)}=-i\left\{Q_{b}^{(2)}, Q_{b}^{(2)}\right\}=0 \Rightarrow\left[Q_{b}^{(2)}\right]^{2}=0
$$

where we have directly applied the BRST symmetry transformation (13) on the expression for $Q_{b}^{(2)}$ (cf. Equation (30)) for the computation of the 1.h.s. of Equation (31). We would like to lay emphasis on the fact that Equation (31) is nothing but the standard relationship between the continuous symmetry transformation $s_{b}$ and its generator $Q_{b}^{(2)}$. The latter is, to be precise, the conserved BRST charge which is the generator of the symmetry transformations (13). We, ultimately, note that the off-shell nilpotency $\left(\left[Q_{b}^{(2)}\right]^{2}=0\right)$ of the $Q_{b}^{(2)}$ has been proven in (31) where we have not used any EL-EOMs and/or CF-type restriction.

Let us now concentrate on the proof of the off-shell nilpotency of the anti-BRST charge $\left(Q_{a b}\right)$. For this purpose, we use the following EL-EOMs:

$$
\begin{aligned}
\dot{\bar{b}} & =\frac{1}{2}\left(p^{2}-m^{2}\right)-2(\dot{\bar{\beta}} \beta-\gamma \chi), \\
p_{\mu} \psi^{\mu}-m \psi_{5} & =2 i e \gamma-i m \gamma-2(\beta \dot{\bar{c}}-\bar{\beta} \dot{c}),
\end{aligned}
$$

that emerge out from the Lagrangian $L_{\bar{b}}$ (when we consider the variables $e$ and $\chi$ for their derivation) to recast the Noether conserved charge $Q_{a b}^{(1)}$ as

$$
\begin{aligned}
Q_{a b}^{(2)}= & \dot{\bar{b}} \bar{c}-\bar{b} \dot{\bar{c}}+2 i e \bar{\beta} \gamma-2 \beta \bar{\beta} \dot{\bar{c}}+2 \bar{c}(\dot{\bar{\beta}} \beta-\gamma \chi)-2 \bar{b} \bar{\beta} \chi \\
& -2 i m \bar{\beta} \gamma+\bar{\beta}^{2} \dot{c}-2 \beta \bar{\beta}^{2} \chi
\end{aligned}
$$

where the superscript (2) on the anti-BRST charge $Q_{a b}^{(2)}$ denotes the fact that it has been derived from the Noether conserved charge $Q_{a b}^{(1)}$. We apply, at this stage, the antiBRST symmetry transformations (12) directly on the antiBRST charge $Q_{a b}^{(2)}$ to obtain

$$
s_{a b} Q_{a b}^{(2)}=-i\left\{Q_{a b}^{(2)}, Q_{a b}^{(2)}\right\}=0 \Rightarrow\left[Q_{a b}^{(2)}\right]^{2}=0
$$

The above observation proves the off-shell nilpotency of the anti-BRST charge $Q_{a b}^{(2)}$ because we do not use EL-EOMs and/or CF-type restriction in its proof. In Equation (34), we have used the basic principle behind the continuous symmetries and their generators. There are other ways, too, to prove the off-shell nilpotency $\left(\left[Q_{(a) b}^{(2)}\right]^{2}=0\right)$ of the (anti-)BRST charges $Q_{(a) b}^{(2)}$. However, we have concentrated, in our present endeavor, only on the standard relationship between the continuous symmetries and their generators.
A couple of decisive features of the BRST formalism is the validity of the off-shell/on-shell nilpotency and absolute anticommutativity properties of the (anti-)BRST symmetries as well as the (anti-)BRST charges. We concentrate now on the proof of the absolute anticommutativity of the conserved and nilpotent (anti-)BRST charges $Q_{(a) b}^{(2)}$. Toward this goal in mind, we first concentrate on the expression for $Q_{b}^{(2)}$ (cf. Equation (30)). Applying directly the anti-BRST symmetry transformations (12) on it, we obtain the following:

$s_{a b} Q_{b}^{(2)}=i(b+\bar{b}+2 \beta \bar{\beta})[\dot{\bar{b}}+2 \chi \gamma+2 \beta \dot{\bar{\beta}}]-i \bar{b} \frac{d}{d \tau}[b+\bar{b}+2 \beta \bar{\beta}]$.

In the terminology of the standard relationship between the continuous symmetry transformation $\left(s_{a b}\right)$ and its generator $Q_{a b}^{(2)}$, it is evident that the 1.h.s. of Equation (35) can be written in an explicit fashion as

$$
s_{a b} Q_{b}^{(2)}=-i\left\{Q_{b}^{(2)}, Q_{a b}^{(2)}\right\} .
$$

A close look at (35) and (36) demonstrates that the absolute anticommutativity of the conserved (anti-)BRST charges (that are off-shell nilpotent of order two) is true if and only if the CF restriction: $b+\bar{b}+2 \beta \bar{\beta}=0$, is imposed on the theory from outside. However, as discussed earlier, this restriction, on the quantum theory, is a physical condition because this CF-type restriction is an (anti-)BRST invariant quantity.

Let us now focus on the expression for the off-shell nilpotent $\left[\left(Q_{a b}^{(2)}\right)^{2}=0\right]$ anti-BRST charge $\left(Q_{a b}^{(2)}\right)$ in Equation (33). The direct application of the BRST symmetry transformation $\left(s_{b}\right)$ of Equation (13) on the anti-BRST charge $Q_{a b}^{(2)}$ in (33) yields the following:

$s_{b} Q_{a b}^{(2)}=i b \frac{d}{d \tau}[b+\bar{b}+2 \beta \bar{\beta}]-i(b+\bar{b}+2 \beta \bar{\beta})[\dot{b}+2 \dot{\beta} \bar{\beta}+2 \gamma \chi]$.

It is straightforward to note that the r.h.s. of (37) would be equal to zero if we impose the (anti-)BRST invariant CFtype restriction $(b+\bar{b}+2 \beta \bar{\beta}=0)$ from outside. Exploiting the beauty of the standard relationship between continuous symmetry transformation $\left(s_{b}\right)$ and its generator (conserved and nilpotent BRST charge $Q_{b}^{(2)}$ ), we note that the l.h.s. of the above equation can be written as

$$
s_{b} Q_{a b}^{(2)}=-i\left\{Q_{a b}^{(2)}, Q_{b}^{(2)}\right\}=0
$$

provided, as stated earlier, we confine ourselves on the submanifold of the quantum Hilbert space of variables where the CF-type restriction $(b+\bar{b}+2 \beta \bar{\beta}=0)$ is satisfied. We have been able to establish an intimate connection between the CF-type restriction and the geometrical objects called gerbes $[5,6]$. The existence of this restriction provides an 
independent identity to the BRST and anti-BRST symmetries and the corresponding (anti-)BRST charges.

We end this subsection with the following remarks. First and foremost, the existence of CF-type restriction is the hallmark of a quantum theory described within the framework of BRST formalism $[5,6]$. Second, the CF-type restriction is responsible for the existence of the coupled (but equivalent) Lagrangians $L_{b}$ and $L_{\bar{b}}$. Third, the absolute anticommutativity of the (anti-)BRST symmetries and corresponding (anti)BRST charges owe their origins to the CF-type restriction. Finally, we have been able to show that $L_{b}$ and $L_{\bar{b}}$ both respect both the (anti-)BRST symmetries due to the existence of CFtype restriction.

\section{BRST Symmetry Transformations: ACSA}

We exploit the basic tenets of ACSA to BRST formalism to derive the proper off-shell nilpotent BRST symmetry transformation (13) where we take into account the antichiral supervariables (defined on the $(1,1)$-dimensional antichiral super submanifold of the general $(1,2)$-dimensional supermanifold). The above antichiral supervariables are the generalizations of the ordinary variables of Lagrangian $L_{b}$ and $\bar{b}(\tau)$ as follows:

$$
\begin{aligned}
x_{\mu}(\tau) \rightarrow X_{\mu}(\tau, \bar{\theta}) & =x_{\mu}(\tau)+\bar{\theta} R_{\mu}^{(1)}(\tau), \\
p_{\mu}(\tau) \rightarrow P_{\mu}(\tau, \bar{\theta}) & =p_{\mu}(\tau)+\bar{\theta} R_{\mu}^{(2)}(\tau), \\
e(\tau) \rightarrow E(\tau, \bar{\theta}) & =e(\tau)+\bar{\theta} f_{1}(\tau), \\
c(\tau) \rightarrow F(\tau, \bar{\theta}) & =c(\tau)+\bar{\theta} b_{1}(\tau), \\
\bar{c}(\tau) \rightarrow \bar{F}(\tau, \bar{\theta}) & =\bar{c}(\tau)+\bar{\theta} b_{2}(\tau), \\
\beta(\tau) \rightarrow \tilde{\beta}(\tau, \bar{\theta}) & =\beta(\tau)+\bar{\theta} f_{2}(\tau), \\
\bar{\beta}(\tau) \rightarrow \bar{\beta}(\tau, \bar{\theta}) & =\bar{\beta}(\tau)+\bar{\theta} f_{3}(\tau), \\
\psi_{\mu}(\tau) \rightarrow \Psi_{\mu}(\tau, \bar{\theta}) & =\psi_{\mu}(\tau)+\bar{\theta} b_{3}(\tau), \\
\psi_{5}(\tau) \rightarrow \Psi_{5}(\tau, \bar{\theta}) & =\psi_{5}(\tau)+\bar{\theta} b_{4}(\tau), \\
\chi(\tau) \rightarrow \tilde{\chi}(\tau, \bar{\theta}) & =\chi(\tau)+\bar{\theta} b_{5}(\tau), \\
\bar{b}(\tau) \rightarrow \bar{B}(\tau, \bar{\theta}) & =\bar{b}(\tau)+\bar{\theta} f_{5}(\tau) \\
\gamma(\tau) \rightarrow \Gamma(\tau, \bar{\theta}) & =\gamma(\tau)+\bar{\theta} b_{6}(\tau), \\
\bar{\beta}(\tau) \rightarrow B(\tau, \bar{\theta}) & =b(\tau)+\bar{\theta} f_{4}(\tau),
\end{aligned}
$$

In the above, we have taken the super expansions along the Grassmannian $\bar{\theta}$-direction of the antichiral $(1,1)$ -dimensional super submanifold which is parameterized by the superspace coordinates $(\tau, \bar{\theta})$. We note that, in the above super expansions, the secondary variables $\left(R_{\mu}^{(1)}, R_{\mu}^{(2)}, f_{1}, f_{2}\right.$, $\left.f_{3}, f_{4}, f_{5}\right)$ are fermionic and the rest of the secondary variables $\left(b_{1}, b_{2}, b_{3}, b_{4}, b_{5}, b_{6}\right)$ are bosonic in nature due to the fermionic $\left(\bar{\theta}^{2}=0\right)$ nature of the Grassmannian variable $\bar{\theta}$. It is elementary to state that, in the limit $\bar{\theta}=0$, we retrieve ordinary variables of our theory described by the Lagrangian $L_{b}$ and $\bar{b}(\tau)$.

The trivial BRST invariant quantities: $s_{b} p_{\mu}=0, s_{b} \gamma=0, s_{b}$ $\beta=0, s_{b} b=0$, imply that the secondary variables $R_{\mu}^{(2)}=b_{6}=$ $f_{2}=f_{4}=0$. This is due to the fact that the basic tenets of ACSA require that the BRST invariant quantities should be independent of the Grassmannian variable $\bar{\theta}$ (which is a mathematical artifact in the superspace formalism). In other words, we have the following:

$$
\begin{aligned}
& P_{\mu}^{(b)}(\tau, \theta)=p_{\mu}(\tau)+\bar{\theta}(0) \equiv p_{\mu}(\tau)+\bar{\theta}\left(s_{b} p_{\mu}(\tau)\right) \Rightarrow P_{\mu}^{(b)}(\tau, \theta)=p_{\mu}(\tau), \\
& \Gamma^{(b)}(\tau, \theta)=\gamma(\tau)+\bar{\theta}(0) \equiv \gamma(\tau)+\bar{\theta}\left(s_{b} \gamma(\tau)\right) \Rightarrow \Gamma^{(b)}(\tau, \theta)=\gamma(\tau), \\
& B^{(b)}(\tau, \theta)=b(\tau)+\bar{\theta}(0) \equiv b(\tau)+\bar{\theta}\left(s_{b} b(\tau)\right) \Rightarrow B^{(b)}(\tau, \theta)=b(\tau), \\
& \tilde{\beta}^{(b)}(\tau, \theta)=\beta(\tau)+\bar{\theta}(0) \equiv \beta(\tau)+\bar{\theta}\left(s_{b} \beta(\tau)\right) \Rightarrow \tilde{\beta}^{(b)}(\tau, \theta)=\beta(\tau),
\end{aligned}
$$

where the superscript $(b)$ on the antichiral supervariables denotes the supervariables that have been obtained after the application of the BRST invariant $\left(s_{b} p_{\mu}=s_{b} \gamma=s_{b} b=s_{b} \beta=0\right)$ restrictions so that the coefficients of $\bar{\theta}$, in the expansions (39), becomes zero. This is due to the fact that there is a mapping (i.e., $s_{b} \leftrightarrow \partial_{\bar{\theta}}, \quad s_{a b} \leftrightarrow \partial_{\theta}$ ) between the (anti-)BRST symmetry transformations $\left(s_{(a) b}\right)$ and the translational operators $\left(\partial_{\theta}, \partial_{\bar{\theta}}\right)$ along the Grassmannian directions of the $(1,2)$ dimensional supermanifold that has been established in Refs. [10-12]. It is crystal clear, from our discussions in this paragraph, that we have to determine precisely all the secondary variables in terms of the basic and auxiliary variables of our theory so that we could know the coefficients of $\bar{\theta}$ in the super expansions (39).

Against the backdrop of our earlier discussions, we have to obtain the precise expressions for the secondary variables so that we could obtain the BRST symmetry transformations $\left(s_{b}\right)$ as the coefficient of $\bar{\theta}$ in the antichiral super expansions (39). Toward this goal in our mind, we have to find out the specific combinations of the nontrivial quantities that are BRST invariant. In this context, we note that the following useful and interesting quantities are BRST invariant, namely,

$$
\begin{aligned}
s_{b}(\bar{\beta} \gamma) & =0, \\
s_{b}(\dot{c}+2 \beta \chi) & =0, \\
s_{b}(e \gamma \chi+e \bar{\beta} \dot{\beta}-i \bar{\beta} \dot{c} \chi) & =0, \\
s_{b}\left(\beta^{2} \bar{\beta}+c \gamma\right) & =0,
\end{aligned}
$$




$$
\begin{aligned}
s_{b}\left(c p_{\mu}+\beta \psi_{\mu}\right) & =0, \\
s_{b}\left(\beta x_{\mu}-i c \psi_{\mu}\right) & =0, \\
s_{b}(b \bar{\beta}+\gamma \bar{c}) & =0, \\
s_{b}(\bar{b}+2 \beta \bar{\beta}) & =0, \\
s_{b}\left(\dot{\psi}_{5}-\chi m\right) & =0 .
\end{aligned}
$$

The basic tenets of ACSA to BRST formalism require that the above quantities, at the quantum level, should be independent of the Grassmannian variable $(\bar{\theta})$ when these are generalized onto the $(1,1)$-dimensional antichiral super submanifold of the general $(1,2)$-dimensional supermanifold. As a consequence, we have the following restrictions on the specific combinations of the antichiral supervariables, namely,

$$
\begin{gathered}
\tilde{\bar{\beta}}(\tau, \bar{\theta}) \Gamma^{(b)}(\tau, \bar{\theta})=\bar{\beta}(\tau) \gamma(\tau), \\
\dot{F}(\tau, \bar{\theta})+2 \tilde{\beta}^{(b)}(\tau, \bar{\theta}) \tilde{\chi}(\tau, \bar{\theta})=\dot{c}(\tau)+2 \beta(\tau) \chi(\tau), \\
E(\tau, \bar{\theta}) \Gamma^{(b)}(\tau, \bar{\theta}) \tilde{\chi}(\tau, \bar{\theta})+E(\tau, \bar{\theta}) \tilde{\bar{\beta}}(\tau, \bar{\theta}) \dot{\tilde{\beta}}^{(b)}(\tau, \bar{\theta}) \\
-i \tilde{\bar{\beta}}(\tau, \bar{\theta}) \dot{F}(\tau, \bar{\theta}) \tilde{\chi}(\tau, \bar{\theta}) \\
=e(\tau) \gamma(\tau) \chi(\tau)+e(\tau) \bar{\beta}(\tau) \dot{\beta}(\tau)-i \bar{\beta}(\tau) \dot{c}(\tau) \chi(\tau), \\
\tilde{\beta}^{2(b)}(\tau, \bar{\theta}) \tilde{\bar{\beta}}(\tau, \bar{\theta})+F(\tau, \bar{\theta}) \Gamma^{(b)}(\tau, \bar{\theta})=\beta^{2}(\tau) \bar{\beta}(\tau)+c(\tau) \gamma(\tau), \\
F(\tau, \bar{\theta}) P_{\mu}^{(b)}(\tau, \bar{\theta})+\tilde{\beta}^{(b)}(\tau, \bar{\theta}) \Psi_{\mu}(\tau, \bar{\theta})=c(\tau) p_{\mu}(\tau)+\beta(\tau) \psi_{\mu}(\tau), \\
\tilde{\beta}^{(b)}(\tau, \bar{\theta}) X_{\mu}(\tau, \bar{\theta})-\mathrm{iF}(\tau, \bar{\theta}) \Psi_{\mu}(\tau, \bar{\theta})=\beta(\tau) x_{\mu}(\tau)-i c(\tau) \psi_{\mu}(\tau), \\
B^{(b)}(\tau, \bar{\theta}) \tilde{\bar{\beta}}(\tau, \bar{\theta})+\Gamma^{(b)}(\tau, \bar{\theta}) \bar{F}(\tau, \bar{\theta})=b(\tau) \bar{\beta}(\tau)+\gamma(\tau) \bar{c}(\tau), \\
\bar{B}(\tau, \bar{\theta})+2 \tilde{\beta}^{(b)}(\tau, \bar{\theta}) \tilde{\bar{\beta}}(\tau, \bar{\theta})=\bar{b}(\tau)+2 \beta(\tau) \bar{\beta}(\tau), \\
\dot{\Psi}{ }_{5}(\tau, \bar{\theta})-\tilde{\chi}(\tau, \bar{\theta}) m=\dot{\psi}_{5}(\tau)-\chi(\tau) m . \quad(42)
\end{gathered}
$$

The above restrictions are quantum gauge (i.e., BRST) invariant conditions on the antichiral supervariables where the supervariables with superscript $(b)$ have been derived and explained in Equation (40) that corresponds to the trivial BRST symmetry transformations.

The substitutions of the antichiral super expansions (39) and the trivial expansions (40) into (42) lead to the following precise expressions for the secondary variables in terms of the basic and auxiliary variables of the coupled (but equivalent)
(anti-)BRST invariant Lagrangians $L_{b}$ and $L_{\bar{b}}$ (cf. Equations (17) and (18)), namely,

$$
\begin{aligned}
R_{\mu}^{(1)} & =c p_{\mu}+\beta \psi_{\mu}, \\
f_{1} & =\dot{c}+2 \beta \chi, \\
b_{1} & =i \beta^{2}, \\
b_{2} & =i b, \\
f_{3} & =i \gamma, \\
b_{3} & =i \beta p_{\mu}, \\
b_{4} & =i \beta m, \\
b_{5} & =i \dot{\beta}, \\
f_{5} & =-2 i \beta \gamma .
\end{aligned}
$$

Ultimately, we obtain the super expansions of (39) in terms of the off-shell nilpotent $\left(s_{b}^{2}=0\right)$ BRST transformations (13) of our theory as follows:

$$
\begin{aligned}
& X_{\mu}^{(b)}(\tau, \bar{\theta})=x_{\mu}+\bar{\theta}\left(c p_{\mu}+\beta \psi_{\mu}\right) \equiv x_{\mu}(\tau)+\bar{\theta}\left(s_{b} x_{\mu}\right), \\
& E^{(b)}(\tau, \bar{\theta})=e(\tau)+\bar{\theta}(\dot{c}+2 \beta \chi) \equiv e(\tau)+\bar{\theta}\left(s_{b} e\right), \\
& F^{(b)}(\tau, \bar{\theta})=c(\tau)+\bar{\theta}\left(i \beta^{2}\right) \equiv c(\tau)+\bar{\theta}\left(s_{b} c\right), \\
& \bar{F}^{(b)}(\tau, \bar{\theta})=\bar{c}(\tau)+\bar{\theta}(i b) \equiv \bar{c}(\tau)+\bar{\theta}\left(s_{b} \bar{c}\right), \\
& \widetilde{\bar{\beta}}^{(b)}(\tau, \bar{\theta})=\bar{\beta}(\tau)+\bar{\theta}(i \gamma) \equiv \bar{\beta}(\tau)+\bar{\theta}\left(s_{b} \bar{\beta}\right), \\
& \Psi_{\mu}^{(b)}(\tau, \bar{\theta})=\psi_{\mu}(\tau)+\bar{\theta}\left(i \beta p_{\mu}\right) \equiv \psi_{\mu}(\tau)+\bar{\theta}\left(s_{b} \psi_{\mu}\right), \\
& \Psi_{5}^{(b)}(\tau, \bar{\theta})=\psi_{5}(\tau)+\bar{\theta}(i \beta m) \equiv \psi_{5}(\tau)+\bar{\theta}\left(s_{b} \psi_{5}\right), \\
& \tilde{\chi}^{(b)}(\tau, \bar{\theta})=\chi(\tau)+\bar{\theta}(i \dot{\beta}) \equiv \chi(\tau)+\bar{\theta}\left(s_{b} \chi\right), \\
& \bar{B}^{(b)}(\tau, \bar{\theta})=\bar{b}(\tau)+\bar{\theta}(-2 i \beta \gamma) \equiv \bar{b}(\tau)+\bar{\theta}\left(s_{b} \bar{b}\right),
\end{aligned}
$$

which are besides the super expansions in (40) (that determine the trivial BRST symmetry transformations as $s_{b} p_{\mu}=0$ , $\left.s_{b} \gamma=0, s_{b} \beta=0, s_{b} b=0\right)$. The superscript $(b)$ on the antichiral supervariable on the l.h.s. of the above expansions denotes the fact that these supervariables have been determined after the quantum gauge (i.e., BRST) invariant restrictions have been imposed on the supervariables as quoted in Equation (42). In our Appendix C, we collect the step-bystep computations that lead to the derivation of (43) from (42). At the classical level, we know that the gauge invariant quantities (GIRs) are physical objects. Within the framework of BRST formalism, all the (anti-)BRST invariant quantities are physical objects at the quantum level. Hence, these quantities should be independent of the Grassmannian variables $(\theta, \bar{\theta})$. In fact, this requirement is one of the basic tenets of ACSA to BRST formalism which is quite physical. 
We end this section with the following remarks. First of all, we note that the coefficients of $\bar{\theta}$ in the super expansions (40) and (44) are nothing but the BRST transformations (13). Second, it is evident that $\partial_{\bar{\theta}} \Omega^{(b)}(x, \bar{\theta})=s_{b} \omega(\tau)$ where $\Omega^{(b)}(x$, $\bar{\theta})$ is the generic antichiral supervariable that is located on the l.h.s. of Equations (40) and (44) and the symbol $\omega(\tau)$ corresponds to the generic ordinary variable that is present in the Lagrangians $L_{b}$ and $L_{\bar{b}}$. Finally, we observe that, due to the mapping $s_{b} \leftrightarrow \partial_{\bar{\theta}}$, the off-shell nilpotency $\left(s_{b}^{2}=0\right)$ of the BRST symmetry transformations (13) is deeply connected with the nilpotency $\left(\partial_{\bar{\theta}}^{2}=0\right)$ of the translational generator $\left(\partial_{\bar{\theta}}\right)$ along the $\bar{\theta}$-direction of $(1,1)$-dimensional antichiral super submanifold on which the antichiral supervariables are defined. It will be noted that only the BRST symmetry transformations have been mentioned in Ref. [33] for the spinning relativistic particle. However, the full set of (anti-)BRST symmetry transformations and the corresponding (anti-)BRST invariant CF-type restriction have been derived in our earlier work [25].

\section{Anti-BRST Symmetry \\ Transformations: ACSA}

In this section, we derive the anti-BRST symmetry transformations (12) by exploiting the theoretical potential and power of ACSA to BRST formalism. Toward this objective in mind, first of all, we generalize the ordinary variables of $L_{\bar{b}}$ (and the auxiliary variable $b(\tau)$ ) onto $(1,1)$-dimensional chiral super submanifold of the general $(1,2)$-dimensional supermanifold (on which our 1D ordinary theory is generalized) as

$$
\begin{aligned}
x_{\mu}(\tau) & \rightarrow X_{\mu}(\tau, \theta)=x_{\mu}(\tau)+\theta \bar{R}_{\mu}^{(1)}(\tau), \\
p_{\mu}(\tau) \rightarrow P_{\mu}(\tau, \theta) & =p_{\mu}(\tau)+\theta \bar{R}_{\mu}^{(2)}(\tau), \\
e(\tau) & \rightarrow E(\tau, \theta)=e(\tau)+\theta \bar{f}_{1}(\tau), \\
c(\tau) & \rightarrow F(\tau, \theta)=c(\tau)+i \theta \bar{b}_{1}(\tau), \\
\bar{c}(\tau) & \rightarrow \bar{F}(\tau, \theta)=\bar{c}(\tau)+i \theta \bar{b}_{2}(\tau), \\
\beta(\tau) & \rightarrow \tilde{\beta}(\tau, \theta)=\beta(\tau)+\theta \bar{f}_{2}(\tau), \\
\bar{\beta}(\tau) \rightarrow \tilde{\beta}(\tau, \theta) & =\bar{\beta}(\tau)+\theta \bar{f}_{3}(\tau), \\
\psi_{\mu}(\tau) \rightarrow \Psi_{\mu}(\tau, \theta) & =\psi_{\mu}(\tau)+\theta \bar{b}_{3}(\tau), \\
\psi_{5}(\tau) \rightarrow \Psi_{5}(\tau, \theta) & =\psi_{5}(\tau)+\theta \bar{b}_{4}(\tau), \\
\chi(\tau) & \rightarrow \tilde{\chi}(\tau, \theta)=\chi(\tau)+\theta \bar{b}_{5}(\tau), \\
\gamma(\tau) & \rightarrow \Sigma(\tau, \theta)=\gamma(\tau)+\theta \bar{b}_{6}(\tau), \\
b(\tau) \rightarrow B(\tau, \theta) & =b(\tau)+\theta \bar{f}_{4}(\tau), \\
\bar{b}(\tau) \rightarrow \bar{B}(\tau, \theta) & =\bar{b}(\tau)+\theta \bar{f}_{5}(\tau),
\end{aligned}
$$

where the $(1,1)$-dimensional chiral super submanifold is parameterized by the superspace coordinates $(\tau, \theta)$ and all the chiral supervariables on the l.h.s. of (45) are a function of these superspace coordinates. The fermionic $\left(\theta^{2}=0\right)$ nature of the Grassmannian variable $\theta$ implies that the secondary variables $\left(\bar{R}_{\mu}^{(1)}, \bar{R}_{\mu}^{(2)}, \bar{f}_{1}, \bar{f}_{2}, \bar{f}_{3}, \bar{f}_{4}, \bar{f}_{5}\right)$ are fermionic and $\left(\bar{b}_{1}, \bar{b}_{2}\right.$, $\left.\bar{b}_{3}, \bar{b}_{4}, \bar{b}_{5}, \bar{b}_{6}\right)$ are bosonic in nature. It is straightforward to note that, in the limit $\theta=0$, we retrieve our ordinary variables of Lagrangian $L_{\bar{b}}$ and the variable $b(\tau)$.

We note that there are trivially anti-BRST invariant quantities (cf. Equation (12)) such as $s_{a b} p_{\mu}=0, s_{a b} \gamma=0$, $s_{a b} \bar{b}=0, s_{a b} \bar{\beta}=0$. As a consequence, we have the following trivial chiral super expansions (with inputs: $\bar{R}_{\mu}^{(2)}=\bar{b}_{6}=\bar{f}_{5}=$ $\bar{f}_{3}=0$ ), namely,

$$
\begin{aligned}
& p_{\mu}(\tau) \rightarrow P_{\mu}^{(a b)}(\tau, \theta)=p_{\mu}(\tau)+\theta(0) \equiv p_{\mu}(\tau)+\theta\left(s_{a b} p_{\mu}(\tau)\right), \\
& \gamma(\tau) \rightarrow \Gamma^{(a b)}(\tau, \theta)=\gamma(\tau)+\theta(0) \equiv \gamma(\tau)+\theta\left(s_{a b} \gamma(\tau)\right), \\
& \bar{b}(\tau) \rightarrow \bar{B}^{(a b)}(\tau, \theta)=\bar{b}(\tau)+\theta(0) \equiv \bar{b}(\tau)+\theta\left(s_{a b} \bar{b}(\tau)\right), \\
& \bar{\beta}(\tau) \rightarrow \widetilde{\bar{\beta}}^{(a b)}(\tau, \theta)=\bar{\beta}(\tau)+\theta(0) \equiv \bar{\beta}(\tau)+\theta\left(s_{a b} \bar{\beta}(\tau)\right),
\end{aligned}
$$

where the superscript $(a b)$ on the supervariables denotes the chiral supervariables where the coefficient of $\theta$ yields the anti-BRST symmetry transformations (12) in view of the mapping: $s_{a b} \leftrightarrow \partial_{\theta}$ [10-12], which becomes transparent when we observe that $\partial_{\theta} \Omega^{(a b)}(\tau, \theta)=s_{a b} \omega(\tau)$ for the generic supervariable $\Omega^{(a b)}(\tau, \theta)$ and the corresponding ordinary generic variable $\omega(\tau)$. The trivial super expansion (46) would be utilized in our further discussions.

The basic ingredient of the ACSA to BRST formalism requires that the nontrivial anti-BRST invariant quantities must be independent of the Grassmannian variable $\theta$ when these quantities are generalized onto the $(1,1)$-dimensional chiral super submanifold. We exploit this idea to determine the secondary variables of the super expansion (45) in terms of the basic and auxiliary variables of $L_{\bar{b}}$. Toward this aim in our mind, we note that the following anti-BRST invariant quantities:

$$
\begin{aligned}
s_{a b}(\beta \gamma) & =0, \\
s_{a b}(\dot{\bar{c}}+2 \bar{\beta} \chi) & =0, \\
s_{a b}(e \gamma \chi-e \dot{\bar{\beta}} \beta+i \dot{\bar{c}} \chi) & =0, \\
s_{a b}\left(\beta \bar{\beta}^{2}-\bar{c} \gamma\right) & =0, \\
s_{a b}\left(\bar{c} p_{\mu}+\bar{\beta} \psi_{\mu}\right) & =0, \\
s_{a b}\left(\bar{\beta} x_{\mu}-i \bar{c} \psi_{\mu}\right) & =0, \\
s_{a b}(\bar{b} \beta-\gamma c) & =0, \\
s_{a b}(b+2 \bar{\beta} \beta) & =0, \\
s_{a b}\left(\dot{\psi}_{5}-\chi m\right) & =0,
\end{aligned}
$$


are found to be very useful and interesting because their generalizations onto the $(1,1)$-dimensional chiral super submanifold, namely,

$$
\begin{aligned}
& \tilde{\beta}(\tau, \theta) \Gamma^{(a b)}(\tau, \theta)=\beta(\tau) \gamma(\tau), \\
& \dot{\bar{F}}(\tau, \theta)+2 \widetilde{\bar{\beta}}^{(a b)}(\tau, \theta) \tilde{\chi}(\tau, \theta)=\dot{\bar{c}}(\tau)+2 \bar{\beta}(\tau) \chi(\tau), \\
& E(\tau, \theta) \Gamma^{(a b)}(\tau, \theta) \tilde{\chi}(\tau, \theta)-E(\tau, \theta) \dot{\tilde{\bar{\beta}}}(\tau, \theta) \tilde{\beta}^{(a b)}(\tau, \theta) \\
& +i \tilde{\beta}(\tau, \theta) \dot{\bar{F}}(\tau, \theta) \tilde{\chi}(\tau, \theta) \\
& =e(\tau) \gamma(\tau) \chi(\tau)-e(\tau) \dot{\bar{\beta}}(\tau) \beta(\tau)+i \beta(\tau) \dot{\bar{c}}(\tau) \chi(\tau), \\
& \tilde{\beta}(\tau, \theta) \widetilde{\bar{\beta}}^{2(a b)}(\tau, \theta)-\bar{F}(\tau, \theta) \Gamma^{(a b)}(\tau, \theta)=\beta(\tau) \bar{\beta}^{(2)}(\tau)-\bar{c}(\tau) \gamma(\tau), \\
& \bar{F}(\tau, \theta) P_{\mu}^{(a b)}(\tau, \theta)+\widetilde{\bar{\beta}}^{(a b)}(\tau, \theta) \Psi_{\mu}(\tau, \theta)=\bar{c}(\tau) p_{\mu}(\tau)+\bar{\beta}(\tau) \psi_{\mu}(\tau) \\
& \widetilde{\bar{\beta}}^{(a b)}(\tau, \theta) X_{\mu}(\tau, \theta)-i \bar{F}(\tau, \theta) \Psi_{\mu}(\tau, \theta)=\bar{\beta}(\tau) x_{\mu}(\tau)-i \bar{c}(\tau) \psi_{\mu}(\tau), \\
& \bar{B}^{(a b)}(\tau, \theta) \tilde{\beta}(\tau, \theta)-\Gamma^{(a b)}(\tau, \theta) F(\tau, \theta)=\bar{b}(\tau) \beta(\tau)-\gamma(\tau) c(\tau), \\
& B(\tau, \theta)+2 \widetilde{\bar{\beta}}^{(a b)}(\tau, \theta) \tilde{\beta}(\tau, \theta)=b(\tau)+2 \bar{\beta}(\tau) \beta(\tau) \\
& \dot{\Psi}_{5}(\tau, \theta)-\tilde{\chi}(\tau, \theta) m=\dot{\psi}_{5}(\tau)-\chi(\tau) m,
\end{aligned}
$$

yield the precise values of the secondary variables of the expansion in (45). To be more precise, we note that the equalities in (48) lead to

$$
\begin{aligned}
\bar{R}_{\mu}^{(1)} & =\bar{c} p_{\mu}+\bar{\beta} \psi_{\mu}, \\
\bar{f}_{1} & =\dot{\bar{c}}+2 \bar{\beta} \chi, \\
\bar{b}_{1} & =i \bar{b}, \\
\bar{b}_{2} & =-i \bar{\beta}^{2}, \\
\bar{f}_{2} & =-i \gamma, \\
\bar{b}_{3} & =i \bar{\beta} p_{\mu}, \\
\bar{b}_{4} & =i \bar{\beta} m, \\
\bar{b}_{5} & =i \dot{\bar{\beta}}, \\
\bar{f}_{4} & =2 i \bar{\beta} \gamma .
\end{aligned}
$$

Thus, we have determined precisely the expressions for the secondary variables in terms of the basic and auxiliary variables of $L_{\bar{b}}$ by requiring that the quantum gauge (i.e., anti-BRST) invariant quantities must be independent of $\theta$ as the Grassmannian variable(s) are only mathematical artifact and they are not physical quantity in the real sense of the word.

The substitutions of all the expressions for the secondary variables (cf. Equation (49)) into the expansions in (45) lead to the following:

$$
\begin{aligned}
& X_{\mu}^{(a b)}(\tau, \theta)=x_{\mu}+\theta\left(\bar{c} p_{\mu}+\bar{\beta} \psi_{\mu}\right) \equiv x_{\mu}(\tau)+\theta\left(s_{a b} x_{\mu}\right), \\
& E^{(a b)}(\tau, \theta)=e(\tau)+\theta(\dot{\bar{c}}+2 \bar{\beta} \chi) \equiv e(\tau)+\theta\left(s_{a b} e\right), \\
& F^{(a b)}(\tau, \theta)=c(\tau)+\theta(i \bar{b}) \equiv c(\tau)+\theta\left(s_{a b} c\right), \\
& \bar{F}^{(a b)}(\tau, \theta)=\bar{c}(\tau)+\theta\left(-i \bar{\beta}^{2}\right) \equiv \bar{c}(\tau)+\theta\left(s_{a b} \bar{c}\right), \\
& \tilde{\beta}^{(a b)}(\tau, \theta)=\bar{\beta}(\tau)+\theta(-i \gamma) \equiv \beta(\tau)+\theta\left(s_{a b} \beta\right), \\
& \Psi_{\mu}^{(a b)}(\tau, \theta)=\psi_{\mu}(\tau)+\theta\left(i \bar{\beta} p_{\mu}\right) \equiv \psi_{\mu}(\tau)+\theta\left(s_{a b} \psi_{\mu}\right), \\
& \Psi_{5}^{(a b)}(\tau, \theta)=\psi_{5}(\tau)+\theta(i \bar{\beta} m) \equiv \psi_{5}(\tau)+\theta\left(s_{a b} \psi_{5}\right), \\
& \tilde{\chi}^{(a b)}(\tau, \theta)=\chi(\tau)+\bar{\theta}(i \dot{\bar{\beta}}) \equiv \chi(\tau)+\theta\left(s_{a b} \chi\right), \\
& B^{(a b)}(\tau, \theta)=b(\tau)+\theta(2 i \bar{\beta} \gamma) \equiv b(\tau)+\theta\left(s_{a b} b\right) .
\end{aligned}
$$

In the above equation, the superscript $(a b)$ on the chiral supervariables (cf. the l.h.s. of (46) and (50)) denotes the super expansions that have been derived after the applications of the anti-BRST invariant restrictions in (48). We note that the coefficients of $\theta$, in the above expansions, are nothing but the anti-BRST symmetry transformations (12) of our 1D system of a massive spinning relativistic particle.

We wrap up this section with the following comments. First and foremost, we observe that the trivial anti-BRST invariant (e.g., $s_{a b} p_{\mu}=s_{a b} \gamma=s_{a b} \bar{b}=s_{a b} \bar{\beta}=0$ ) variables have been incorporated in the super expansions in (46). Second, the nontrivial anti-BRST symmetry transformations (12) have been incorporated in the super expansions (50). Finally, we have exploited the basic idea of ACSA to BRST formalism where we have demanded that the anti-BRST (i.e., quantum gauge) invariant quantities must be independent of the Grassmannian variable $\theta$ when they are generalized onto the $(1,1)$-dimensional chiral super submanifold of the general $(1,2)$-dimensional supermanifold.

\section{Symmetry Invariance of Lagrangians: ACSA}

In this section, we capture the (anti-)BRST symmetry invariance of the coupled (but equivalent) $L_{b}$ and $L_{\bar{b}}$ (cf. Equations (20)-(23)) within the framework of ACSA to BRST formalism. In this context, it is pertinent to point out that the CFtype condition $(b+\bar{b}+2 \beta \bar{\beta}=0)$ is responsible for the existence of the coupled (but equivalent) Lagrangians $L_{b}$ and $L_{\bar{b}}$ and it is also responsible for the absolute anticommutativity (i.e., $\left\{s_{b}, s_{a b}\right\}=0$ ) of the (anti-)BRST symmetries $\left(s_{(a) b}\right)$ and the absolute anticommutativity (i.e., $\left\{Q_{b}, Q_{a b}\right\}=0$ ) of the corresponding conserved $\left(\dot{Q}_{(a) b}=0\right)$ and off-shell nilpotent (i.e., 
$\left.\dot{Q}_{(a) b}^{2}=0\right)$ (anti-)BRST charges $\left(Q_{(a) b}\right)$. Thus, it is important for us to capture the existence of the (anti-)BRST invariant (i.e., $s_{(a) b}[b+\bar{b}+2 \beta \bar{\beta}]=0$ ) CF-type restriction in the context of symmetry considerations of the coupled (but equivalent) Lagrangians $L_{b}$ and $L_{\bar{b}}$ for our 1D system of a reparameterization invariant massive model of spinning relativistic particle.

Against the backdrop of the above statements, first of all, we consider the (anti-)BRST symmetry invariance (cf. Equations (20) and (21)) of the Lagrangians $L_{b}$ and $L_{\bar{b}}$. Toward this goal in mind, we generalize these Lagrangians on the $(1,1)$-dimensional chiral and antichiral super submanifolds (in terms of the corresponding supervariables) as

$$
\begin{aligned}
& L_{\bar{b}} \rightarrow \tilde{L}_{\bar{b}}^{(c)}(\tau, \theta) \\
& =\tilde{L}_{f}^{(c)}(\tau, \theta)+\bar{B}^{(a b)}(\tau, \theta) \bar{B}^{(a b)}(\tau, \theta)-\bar{B}^{(a b)}(\tau, \theta) \\
& \times\left[\dot{E}^{(a b)}(\tau, \theta)-2 \widetilde{\widetilde{\beta}}^{(a b)}(\tau, \theta) \tilde{\beta}^{(a b)}(\tau, \theta)\right]-i \dot{\bar{F}}^{(a b)}(\tau, \theta) \dot{F}^{(a b)}(\tau, \theta) \\
& +\widetilde{\widetilde{\beta}}^{(a b)}(\tau, \theta) \widetilde{\bar{\beta}}^{(a b)}(\tau, \theta) \tilde{\beta}^{(a b)}(\tau, \theta) \tilde{\beta}^{(a b)}(\tau, \theta)+2 i \tilde{\chi}^{(a b)}(\tau, \theta) \\
& \times\left[\tilde{\beta}^{(a b)}(\tau, \theta) \dot{\bar{F}}^{(a b)}(\tau, \theta)-\widetilde{\bar{\beta}}^{(a b)}(\tau, \theta) \dot{F}^{(a b)}(\tau, \theta)\right]+2 E^{(a b)}(\tau, \theta) \\
& \times\left[\dot{\overline{\tilde{\beta}}}^{(a b)}(\tau, \theta) \tilde{\beta}^{(a b)}(\tau, \theta)-\Gamma^{(a b)}(\tau, \theta) \tilde{\chi}^{(a b)}(\tau, \theta)\right]+2 \Gamma^{(a b)}(\tau, \theta) \\
& \times\left[\tilde{\beta}^{(a b)}(\tau, \theta) \bar{F}^{(a b)}(\tau, \theta)-\widetilde{\bar{\beta}}^{(a b)}(\tau, \theta) F^{(a b)}(\tau, \theta)\right]+m \\
& \times\left[\widetilde{\bar{\beta}}^{(a b)}(\tau, \theta) \dot{\tilde{\beta}}^{(a b)}(\tau, \theta)-\dot{\bar{\beta}}^{(a b)}(\tau, \theta) \tilde{\beta}^{(a b)}(\tau, \theta)\right. \\
& \left.\times+\Gamma^{(a b)}(\tau, \theta) \tilde{\chi}^{(a b)}(\tau, \theta)\right]-\dot{\Gamma}^{(a b)}(\tau, \theta) \Psi_{5}^{(a b)}(\tau, \theta), \\
& L_{b} \rightarrow \tilde{L}_{b}^{(a c)}(\tau, \bar{\theta}) \\
& =\tilde{L}_{f}^{(a c)}(\tau, \bar{\theta})+B^{(b)}(\tau, \bar{\theta}) B^{(b)}(\tau, \bar{\theta})+B^{(b)}(\tau, \bar{\theta}) \\
& \times\left[\dot{E}^{(b)}(\tau, \bar{\theta})+2 \widetilde{\bar{\beta}}^{(b)}(\tau, \bar{\theta}) \tilde{\beta}^{(b)}(\tau, \bar{\theta})\right]-i \dot{\bar{F}}^{(b)}(\tau, \bar{\theta}) \dot{F}^{(b)}(\tau, \bar{\theta}) \\
& +\widetilde{\bar{\beta}}^{(b)}(\tau, \bar{\theta}) \widetilde{\bar{\beta}}^{(b)}(\tau, \bar{\theta}) \tilde{\beta}^{(b)}(\tau, \bar{\theta}) \tilde{\beta}^{(b)}(\tau, \bar{\theta})+2 i \tilde{\chi}^{(b)}(\tau, \bar{\theta}) \\
& \times\left[\tilde{\beta}^{(b)}(\tau, \bar{\theta}) \dot{\bar{F}}^{(b)}(\tau, \bar{\theta})-\widetilde{\bar{\beta}}^{(b)}(\tau, \bar{\theta}) \dot{F}^{(b)}(\tau, \bar{\theta})\right]-2 E^{(b)}(\tau, \bar{\theta}) \\
& \times\left[\widetilde{\bar{\beta}}^{(b)}(\tau, \bar{\theta}) \dot{\tilde{\beta}}^{(b)}(\tau, \bar{\theta})+\Gamma^{(b)}(\tau, \bar{\theta}) \tilde{\chi}^{(b)}(\tau, \bar{\theta})\right]+2 \Gamma^{(b)}(\tau, \bar{\theta}) \\
& \times\left[\tilde{\beta}^{(b)}(\tau, \bar{\theta}) \bar{F}^{(b)}(\tau, \bar{\theta})-\widetilde{\bar{\beta}}^{(b)}(\tau, \bar{\theta}) F^{(b)}(\tau, \bar{\theta})\right]+m \\
& \times\left[\widetilde{\bar{\beta}}^{(b)}(\tau, \bar{\theta}) \dot{\tilde{\beta}}^{(b)}(\tau, \bar{\theta})-\dot{\overline{\tilde{\beta}}}^{(b)}(\tau, \bar{\theta}) \tilde{\beta}^{(b)}(\tau, \bar{\theta})\right. \\
& \left.\times+\Gamma^{(b)}(\tau, \bar{\theta}) \tilde{\chi}^{(b)}(\tau, \bar{\theta})\right]-\dot{\Gamma}^{(b)}(\tau, \bar{\theta}) \Psi_{5}^{(b)}(\tau, \bar{\theta}),
\end{aligned}
$$

where the superscripts $(c)$ and $(a c)$ on the super Lagrangians (i.e., $\tilde{L}_{\bar{b}}^{(c)}, \tilde{L}_{b}^{(a c)}$ ) denote that these Lagrangians incorporate chiral and antichiral supervariables that have been obtained after the (anti-)BRST invariant restrictions (cf. Equations
(40), (44), (46), and (50)). Furthermore, we note that the super first-order Lagrangians are

$$
\begin{aligned}
\tilde{L}_{f}^{(c)}(\tau, & \theta) \\
= & P_{\mu}^{(a b)}(\tau, \theta) \dot{X}^{\mu(a b)}(\tau, \theta)-\frac{1}{2} E^{(a b)}(\tau, \theta) \\
& \times\left[P_{\mu}^{(a b)}(\tau, \theta) P^{\mu(a b)}(\tau, \theta)-m^{2}\right] \\
& +\frac{i}{2}\left[\Psi_{\mu}^{(a b)}(\tau, \theta) \dot{\Psi}^{\mu(a b)}(\tau, \theta)-\Psi_{5}^{(a b)}(\tau, \theta) \dot{\Psi}_{5}^{(a b)}(\tau, \theta)\right] \\
& +i \tilde{\chi}^{(a b)}(\tau, \theta)\left[P_{\mu}^{(a b)}(\tau, \theta) \Psi^{\mu(a b)}(\tau, \theta)-m \Psi_{5}^{(a b)}(\tau, \theta)\right], \\
\tilde{L}_{f}^{(a c)}(\tau, \bar{\theta}) & \\
= & P_{\mu}^{(b)}(\tau, \bar{\theta}) \dot{X}^{\mu(b)}(\tau, \bar{\theta})-\frac{1}{2} E^{(b)}(\tau, \bar{\theta}) \\
& \times\left[P_{\mu}^{(b)}(\tau, \bar{\theta}) P^{\mu(b)}(\tau, \bar{\theta})-m^{2}\right] \\
& +\frac{i}{2}\left[\Psi_{\mu}^{(b)}(\tau, \bar{\theta}) \dot{\Psi}^{\mu(b)}(\tau, \bar{\theta})-\Psi_{5}^{(b)}(\tau, \bar{\theta}) \dot{\Psi}_{5}^{(b)}(\tau, \bar{\theta})\right] \\
& +i \tilde{\chi}^{(b)}(\tau, \bar{\theta})\left[P_{\mu}^{(b)}(\tau, \bar{\theta}) \Psi^{\mu(b)}(\tau, \bar{\theta})-m \Psi_{5}^{(b)}(\tau, \bar{\theta})\right] .
\end{aligned}
$$

It is, at this stage, very essential to point out that some of the supervariables are (anti-)BRST invariant and, hence, they are merely ordinary variables. For instance, we note that $P_{\mu}^{(a b)}(\tau, \theta)=P_{\mu}^{(b)}(\tau, \bar{\theta})=p_{\mu}(\tau), \Gamma^{(b)}(\tau, \bar{\theta})=\Gamma^{(a b)}(\tau, \theta)=\gamma(\tau)$, $\widetilde{\bar{\beta}}^{(a b)}(\tau, \theta)=\bar{\beta}(\tau), \tilde{\beta}^{(b)}(\tau, \bar{\theta})=\beta(\tau), \bar{B}^{(a b)}(\tau, \theta)=\bar{b}(\tau), B^{(b)}(\tau$, $\bar{\theta})=b(\tau)$.

In view of the mappings: $s_{b} \leftrightarrow \partial_{\bar{\theta}}, s_{a b} \leftrightarrow \partial_{\theta}$ [10-12], we can now capture the (anti-)BRST invariance of $L_{\bar{b}}$ and $L_{b}$ (cf. Equations (20) and (21)) as

$$
\begin{aligned}
& \frac{\partial}{\partial \bar{\theta}} \tilde{L}_{b}^{(a c)}(\tau, \bar{\theta}) \\
& \quad=\frac{d}{d \tau}\left[\frac{c}{2}\left(p^{2}+m^{2}\right)+\frac{\beta}{2}\left(p_{\mu} \psi^{\mu}+m \psi_{5}\right)+b(\dot{c}+2 \beta \chi)\right] \\
& \quad \equiv s_{b} L_{b}, \\
& \frac{\partial}{\partial \theta} \tilde{L}_{\bar{b}}^{(c)}(\tau, \theta) \\
& \quad=\frac{d}{d \tau}\left[\frac{\bar{c}}{2}\left(p^{2}+m^{2}\right)+\frac{\bar{\beta}}{2}\left(p_{\mu} \psi^{\mu}-m \psi_{5}\right)-\bar{b}(\dot{\bar{c}}+2 \bar{\beta} \chi)\right] \\
& \quad \equiv s_{a b} L_{\bar{b}} .
\end{aligned}
$$

Thus, we have captured the (anti-)BRST invariance of the Lagrangians $L_{\bar{b}}$ and $L_{b}$ (cf. Equations (21) and (20)) within the framework of ACSA to BRST formalism. Geometrically, the chiral super Lagrangian $\tilde{L}_{\bar{b}}^{(c)}(\tau, \theta)$ is a unique sum of the combination of (super)variables that have been obtained after the quantum gauge (i.e., anti-BRST) invariant restrictions. The translation of this unique sum, along the $\theta$ 
-direction of $(1,1)$-dimensional chiral super submanifold, generates a total "time" derivative (cf. Equation (21)) in the ordinary space. As a consequence, the action integral $S=$ $\int_{-\infty}^{+\infty} d \tau L_{\bar{b}}$ remains invariant under the anti-BRST symmetry transformations $\left(s_{a b}\right)$. In exactly similar fashion, we can discuss the BRST invariance of the Lagrangian $L_{b}$ (cf. Equation (20)) within the framework of ACSA to BRST formalism and provide the geometrical interpretation for the super antichiral Lagrangian $\tilde{L}_{b}^{(a c)}(\tau, \bar{\theta})$ and its connection with the BRST-invariance (cf. Equation (20)) in the ordinary space.

Now let us capture Equations (22) and (23), where the BRST symmetry transformation operates on $L_{\bar{b}}$ and the anti-BRST symmetry transformation acts on $L_{b}$, within the purview of ACSA to BRST formalism. In this context, let us, first of all, generalize the Lagrangian $L_{b}$ onto the chiral $(1,1)$-dimensional super submanifold such that chiral supervariables (with the superscript $(a b)$ ) appear in it. In other words, we have the following generalization:

$$
\begin{aligned}
L_{b} \rightarrow & \tilde{L}_{b}^{(c)}(\tau, \theta) \\
= & \tilde{L}_{f}^{(c)}(\tau, \theta)+B^{(a b)}(\tau, \theta) B^{(a b)}(\tau, \theta)+B^{(a b)}(\tau, \theta) \\
& \times\left[\dot{E}^{(a b)}(\tau, \theta)+2 \widetilde{\bar{\beta}}^{(a b)}(\tau, \theta) \tilde{\beta}^{(a b)}(\tau, \theta)\right]-i \dot{\bar{F}}^{(a b)}(\tau, \theta) \dot{F}^{(a b)} \\
& \times(\tau, \theta) \widetilde{\bar{\beta}}^{(a b)}(\tau, \theta) \widetilde{\bar{\beta}}^{(a b)}(\tau, \theta) \tilde{\beta}^{(a b)}(\tau, \theta) \tilde{\beta}^{(a b)}(\tau, \theta)+2 i \tilde{\chi}^{(a b)}(\tau, \theta) \\
& \times\left[\tilde{\beta}^{(a b)}(\tau, \theta) \dot{\bar{F}}^{(a b)}(\tau, \theta)-\widetilde{\bar{\beta}}^{(a b)}(\tau, \theta) \dot{F}^{(a b)}(\tau, \theta)\right]-2 E^{(a b)}(\tau, \theta) \\
& \times\left[\widetilde{\tilde{\beta}}^{(a b)}(\tau, \theta) \dot{\tilde{\beta}}^{(a b)}(\tau, \theta)+\Gamma^{(a b)}(\tau, \theta) \tilde{\chi}^{(a b)}(\tau, \theta)\right]+2 \Gamma^{(a b)}(\tau, \theta) \\
& \times\left[\tilde{\beta}^{(a b)}(\tau, \theta) \bar{F}^{(a b)}(\tau, \theta)-\widetilde{\bar{\beta}}^{(a b)}(\tau, \theta) F^{(a b)}(\tau, \theta)\right]+m \\
& \times\left[\widetilde{\bar{\beta}}^{(a b)}(\tau, \theta) \dot{\tilde{\tilde{\beta}}}^{(b)}(\tau, \theta)-\dot{\overline{\tilde{\beta}}}^{(a b)}(\tau, \theta) \tilde{\tilde{\beta}}^{(a b)}(\tau, \theta)\right. \\
& \left.+\Gamma^{(a b)}(\tau, \theta) \tilde{\chi}^{(a b)}(\tau, \theta)\right]-\dot{\Gamma}^{(a b)}(\tau, \theta) \Psi_{5}^{(a b)}(\tau, \theta) .
\end{aligned}
$$

It should be noted that some of the chiral supervariables with the superscript $(a b)$ are, primarily, the ordinary variables. For instance, we note that all the chiral supervariables on the 1.h.s. of (46) are actually such variables (i.e., $P_{\mu}^{(a b)}(\tau, \theta)=$ $\left.p_{\mu}(\tau), \Gamma^{(a b)}(\tau, \theta)=\gamma(\tau), \widetilde{\bar{\beta}}^{(a b)}(\tau, \theta)=\bar{\beta}(\tau)\right)$. Keeping in our mind the mapping: $s_{a b} \Leftrightarrow \partial_{\theta}$, it is clear that we can operate $\partial_{\theta}$ on the above chiral Lagrangian $\tilde{L}_{b}^{(c)}(\tau, \theta)$ to yield the following:

$$
\begin{aligned}
\frac{\partial}{\partial \theta} \tilde{L}_{b}^{(c)}(\tau, \theta) \\
=\frac{d}{d \tau}\left[\frac{\bar{c}}{2}\left(p^{2}+m^{2}\right)+\frac{\bar{\beta}}{2}\left(p_{\mu} \psi^{\mu}+m \psi_{5}\right)+b(\dot{\bar{c}}+2 \bar{\beta} \chi)+2 i e \bar{\beta} \gamma\right] \\
\quad-(\dot{\bar{c}}+2 \bar{\beta} \chi)\left[\frac{d}{d \tau}(b+\bar{b}+2 \beta \bar{\beta})\right]+(2 i \bar{\beta} \gamma)(b+\bar{b}+2 \beta \bar{\beta}) \\
\equiv s_{a b} L_{b} .
\end{aligned}
$$

The above observation establishes the fact that Lagrangian $L_{b}$ also respects the anti-BRST symmetry transformation (12) provided we invoke the CF-type restriction $(b+\bar{b}+2 \beta \bar{\beta}=0)$ from outside. In other words, we have captured the existence of the (anti-)BRST invariant CF-type restriction within the framework of ACSA to BRST formalism and have proved that the Lagrangian $L_{b}$ (which is perfectly BRST invariant (cf. Equation (20))) is also invariant w.r.t. the anti-BRST symmetry transformation (12) provided we confine ourselves to the submanifold of the quantum variable where the CF-type restriction is satisfied.

At this juncture, we generalize the ordinary Lagrangian $L_{\bar{b}}$ to its counterpart antichiral Lagrangian $\tilde{L}_{\bar{b}}^{(a c)}(\tau, \bar{\theta})$ on the $(1,1)$-dimensional antichiral super submanifold as

$$
\begin{aligned}
L_{\bar{b}} \rightarrow & \tilde{L}_{\bar{b}}^{(a c)}(\tau, \bar{\theta}) \\
= & \tilde{L}_{f}^{(a c)}(\tau, \bar{\theta})+\bar{B}^{(b)}(\tau, \bar{\theta}) \bar{B}^{(b)}(\tau, \bar{\theta})-\bar{B}^{(b)}(\tau, \bar{\theta}) \\
& \times\left[\dot{E}^{(b)}(\tau, \bar{\theta})-2 \widetilde{\bar{\beta}}^{(b)}(\tau, \bar{\theta}) \tilde{\beta}^{(b)}(\tau, \bar{\theta})\right] \\
& -i \dot{\bar{F}}^{(b)}(\tau, \bar{\theta}) \dot{F}^{(b)}(\tau, \bar{\theta})+\widetilde{\bar{\beta}}^{(b)}(\tau, \bar{\theta}) \tilde{\widetilde{\beta}}^{(b)}(\tau, \bar{\theta}) \tilde{\beta}^{(b)} \\
& \times(\tau, \bar{\theta}) \tilde{\beta}^{(b)}(\tau, \bar{\theta})+2 i \tilde{\chi}^{(b)}(\tau, \bar{\theta}) \\
& \times\left[\tilde{\beta}^{(b)}(\tau, \bar{\theta}) \dot{\bar{F}}^{(b)}(\tau, \bar{\theta})-\widetilde{\tilde{\beta}}^{(b)}(\tau, \bar{\theta}) \dot{F}^{(b)}(\tau, \bar{\theta})\right]+2 E^{(b)}(\tau, \bar{\theta}) \\
& \times\left[\dot{\overline{\tilde{\beta}}}^{(b)}(\tau, \bar{\theta}) \tilde{\beta}^{(b)}(\tau, \bar{\theta})-\Gamma^{(b)}(\tau, \bar{\theta}) \tilde{\chi}^{(b)}(\tau, \bar{\theta})\right]+2 \Gamma^{(b)}(\tau, \bar{\theta}) \\
& \times\left[\tilde{\beta}^{(b)}(\tau, \bar{\theta}) \bar{F}^{(b)}(\tau, \bar{\theta})-\widetilde{\tilde{\beta}}^{(b)}(\tau, \bar{\theta}) F^{(b)}(\tau, \bar{\theta})\right]+m \\
& \times\left[\widetilde{\bar{\beta}}^{(b)}(\tau, \bar{\theta}) \dot{\tilde{\beta}}^{(b)}(\tau, \bar{\theta})-\dot{\tilde{\bar{\beta}}}^{(b)}(\tau, \bar{\theta}) \tilde{\beta}^{(b)}(\tau, \bar{\theta})\right. \\
+ & \left.\Gamma^{(b)}(\tau, \bar{\theta}) \tilde{\chi}^{(b)}(\tau, \bar{\theta})\right]-\dot{\Gamma}^{(b)}(\tau, \bar{\theta}) \Psi_{5}^{(b)}(\tau, \bar{\theta}) .
\end{aligned}
$$

It will be noted that some of the above antichiral supervariables (cf. Equation (40)) are basically ordinary variables (e.g., $P_{\mu}^{(b)}(\tau, \bar{\theta})=p_{\mu}(\tau), \quad \tilde{\beta}^{(b)}(\tau, \bar{\theta})=\beta(\tau), \quad \Gamma^{(b)}(\tau, \bar{\theta})=\gamma(\tau)$ ). As far as the dependence on the Grassmannian variable of $\tilde{L}_{b}^{(a c)}(\tau, \bar{\theta})$ is concerned, it is straightforward to note that we have $\bar{\theta}$-dependence. Thus, the mapping: $s_{b} \leftrightarrow \partial_{\bar{\theta}}$, allows us to apply, on the super Lagrangian $\tilde{L}_{\bar{b}}^{(a c)}(\tau, \bar{\theta})$, a derivative $\left(\partial_{\bar{\theta}}\right)$ w.r.t. the Grassmannian variable $\bar{\theta}$. The ensuing mathematical expression, as the outcome of the above operation, is as follows:

$$
\begin{aligned}
\frac{\partial}{\partial \bar{\theta}} \tilde{L}_{b}^{(a c)}(\tau, \bar{\theta}) & \\
= & \frac{d}{d \tau}\left[\frac{c}{2}\left(p^{2}+m^{2}\right)+\frac{\beta}{2}\left(p_{\mu} \psi^{\mu}+m \psi_{5}\right)-\bar{b}(\dot{c}+2 \beta \chi)+2 i e \beta \gamma\right] \\
& +(\dot{c}+2 \beta \chi)\left[\frac{d}{d \tau}(b+\bar{b}+2 \beta \bar{\beta})\right]-(2 i \beta \gamma)(b+\bar{b}+2 \beta \bar{\beta}) \\
\equiv & s_{b} L_{\bar{b}} .
\end{aligned}
$$


At this stage, it is an elementary exercise to state that the perfectly anti-BRST invariant Lagrangian $L_{\bar{b}}$ (cf. Equation (21)) also respects the BRST symmetry transformations (13) provided the whole theory is considered on a submanifold of the Hilbert space of quantum variables where the CF-type restriction $(b+\bar{b}+2 \beta \bar{\beta}=0)$ is satisfied. In other words, the Lagrangian $L_{\bar{b}}$ respects the BRST symmetry transformations (13) provided we impose the CF-type restriction from outside. Thus, we have derived the CF-type restriction (cf. Equation (57)) within the ambit of ACSA to BRST formalism.

We end this section with the remark that the CF-type restriction is the hallmark $[5,6]$ of a quantum theory (discussed within the framework of BRST formalism). We have shown its existence on our theory within the framework of ACSA to BRST formalism. Hence, we have achieved a proper BRST quantization of our theory of the $1 \mathrm{D}$ system.

\section{Off-Shell Nilpotency and Absolute Anticommutativity of the (Anti-)BRST Charges: ACSA}

We have already seen that the (anti-)BRST symmetry transformations (13) and (12) are off-shell nilpotent $\left(s_{(a) b}^{2}=0\right)$ and absolutely anticommuting (cf. Equation (15)) in nature provided the CF-type restriction (cf. Equation (14)) is imposed from outside on our theory. As the off-shell nilpotent (anti-)BRST symmetry transformations are generated by the conserved (anti-)BRST charges, the above off-shell nilpotency and absolute anticommutativity are also respected by the conserved and off-shell nilpotent (anti-)BRST charges. In our present section, we capture these properties of the conserved $\left(\dot{Q}_{(a) b}=0\right)$ fermionic (anti-)BRST charges $Q_{(a) b}$ within the framework of ACSA to BRST formalism.

We have already demonstrated that the Noether conserved charges $Q_{(a) b}^{(1)}$ (cf. Equations (26) and (27)) are not off-shell nilpotent. In fact, they are on-shell nilpotent. Using the EL-EOMs, we have recast these conserved charges into another forms (cf. Equations (30) and (33)) and denoted them by $Q_{(a) b}^{(2)}$. These latter forms of the charges turn out to be offshell nilpotent of order two (i.e., $\left.\left(Q_{(a) b}^{(2)}\right)^{2}=0\right)$. We now concentrate on $Q_{b}^{(2)}$ and generalize the BRST charge $Q_{b}^{(2)}$ onto the $(1,1)$-dimensional antichiral super submanifold as

$$
\begin{aligned}
Q_{b}^{(2)} \rightarrow & \tilde{Q}_{b}^{(2)}(\tau, \bar{\theta}) \\
= & B^{(b)}(\tau, \bar{\theta}) \dot{F}^{(b)}(\tau, \bar{\theta})-\dot{B}^{(b)}(\tau, \bar{\theta}) F^{(b)}(\tau, \bar{\theta})+2 i E^{(b)} \\
& \times(\tau, \bar{\theta}) \tilde{\beta}^{(b)}(\tau, \bar{\theta}) \Gamma^{(b)}(\tau, \bar{\theta})+2 \tilde{\beta}^{(b)}(\tau, \bar{\theta}) \widetilde{\bar{\beta}}^{(b)} \\
& \times(\tau, \bar{\theta}) \dot{F}^{(b)}(\tau, \bar{\theta})-2 F^{(b)}(\tau, \bar{\theta}) \\
& \times\left[\widetilde{\tilde{\beta}}^{(b)}(\tau, \bar{\theta}) \dot{\tilde{\beta}}^{(b)}(\tau, \bar{\theta})+\Gamma^{(b)}(\tau, \bar{\theta}) \tilde{\chi}^{(b)}(\tau, \bar{\theta})\right] \\
& +2 B^{(b)}(\tau, \bar{\theta}) \tilde{\beta}^{(b)}(\tau, \bar{\theta}) \tilde{\chi}^{(b)}(\tau, \bar{\theta})-2 i m \Gamma^{(b)}(\tau, \bar{\theta}) \tilde{\beta}^{(b)}
\end{aligned}
$$

$$
\begin{aligned}
& \times(\tau, \bar{\theta})-\tilde{\beta}^{(b)}(\tau, \bar{\theta}) \tilde{\beta}^{(b)}(\tau, \bar{\theta}) \dot{\bar{F}}^{(b)}(\tau, \bar{\theta}) \\
& +2 \tilde{\chi}^{(b)}(\tau, \bar{\theta}) \tilde{\beta}^{(b)}(\tau, \bar{\theta}) \tilde{\beta}^{(b)}(\tau, \bar{\theta}) \widetilde{\bar{\beta}}^{(b)}(\tau, \bar{\theta}),
\end{aligned}
$$

where all the antichiral supervariables with the superscript $(b)$ have been derived in Equations (40) and (44). It is evident that there are some supervariables in the above expression for $\tilde{Q}_{b}^{(2)}(\tau, \bar{\theta})$ which are actually ordinary variables (cf. Equation (40)). Keeping in our mind the mapping: $s_{b} \leftrightarrow \partial_{\bar{\theta}}$ [10-12], it can be explicitly checked that

$$
\frac{\partial}{\partial \bar{\theta}} \tilde{Q}_{b}^{(2)}(\tau, \bar{\theta}) \equiv \int d \bar{\theta} \tilde{Q}_{b}^{(2)}(\tau, \bar{\theta})=0 \Leftrightarrow s_{b} Q_{b}^{(2)}=0 .
$$

The above relationship is nothing but the proof of the offshell nilpotency $\left[\left(Q_{b}^{(2)}\right)^{2}=0\right]$ of the conserved BRST charge $Q_{b}^{(2)}$. To corroborate this statement, we note that, in the ordinary space, the observation $s_{b} Q_{b}^{(2)}=0$ can be mathematically stated as Equation (31) which proves the off-shell nilpotency $\left[\left(Q_{b}^{(2)}\right)^{2}=0\right]$ of the conserved charge $Q_{b}^{(2)}$.

We now focus on the proof of the nilpotency $\left[\left(Q_{a b}^{(2)}\right)^{2}=0\right]$ of the anti-BRST charge $Q_{a b}^{(2)}$ (cf. Equation (33)) within the ambit of ACSA to BRST formalism. In this context, we note that $Q_{a b}^{(2)}$ can be generalized onto the $(1,1)$-dimensional chiral super submanifold as

$$
\begin{aligned}
Q_{a b}^{(2)} \rightarrow & \tilde{Q}_{a b}^{(2)}(\tau, \theta) \\
= & \dot{\bar{B}}^{(a b)}(\tau, \theta) \bar{F}^{(a b)}(\tau, \theta)-\bar{B}^{(a b)}(\tau, \theta) \dot{\bar{F}}^{(a b)}(\tau, \theta) \\
& +2 i E^{(a b)}(\tau, \theta) \widetilde{\bar{\beta}}^{(a b)}(\tau, \theta) \Gamma^{(a b)}(\tau, \theta)-2 \tilde{\beta}^{(a b)} \\
& \times(\tau, \theta) \widetilde{\bar{\beta}}^{(a b)}(\tau, \theta) \dot{\bar{F}}^{(a b)}(\tau, \theta)+2 \bar{F}^{(a b)}(\tau, \theta) \\
& \times\left[\dot{\overline{\bar{\beta}}}^{(a b)}(\tau, \theta) \tilde{\beta}^{(a b)}(\tau, \theta)-\Gamma^{(a b)}(\tau, \theta) \tilde{\chi}^{(a b)}(\tau, \theta)\right] \\
& -2 \bar{B}^{(a b)}(\tau, \theta) \widetilde{\bar{\beta}}^{(a b)}(\tau, \theta) \tilde{\chi}^{(a b)}(\tau, \theta)-2 i m \Gamma^{(a b)} \\
& \times(\tau, \theta) \widetilde{\bar{\beta}}^{(a b)}(\tau, \theta)+\widetilde{\bar{\beta}}^{(a b)}(\tau, \theta) \widetilde{\bar{\beta}}^{(a b)}(\tau, \theta) \dot{F}^{(a b)}(\tau, \theta) \\
& -2 \tilde{\chi}^{(a b)}(\tau, \theta) \widetilde{\bar{\beta}}^{(a b)}(\tau, \theta) \widetilde{\bar{\beta}}^{(a b)}(\tau, \theta) \tilde{\beta}^{(a b)}(\tau, \theta),
\end{aligned}
$$

where all the supervariables with superscript $(a b)$ are chiral expansions that have been quoted in Equations (46) and (50). It is pertinent to point out that some of the chiral supervariables, on the r.h.s. of (60), are actually ordinary variables (cf. Equation (46)) because they are anti-BRST invariant (e.g., $\left.s_{a b} \bar{\beta}=s_{a b} \bar{b}=s_{a b} p_{\mu}=0\right)$ variables. In view of the mapping: $s_{a b} \leftrightarrow \partial_{\theta}$ [10-12], we are in the position to operate a 
derivative w.r.t. the Grassmannian variable $\theta$ on the expression for the super anti-BRST charge to show that

$$
\frac{\partial}{\partial \theta} \tilde{Q}_{a b}^{(2)}(\tau, \theta) \equiv \int d \theta \tilde{Q}_{a b}^{(2)}(\tau, \theta)=0 \Leftrightarrow s_{a b} Q_{a b}^{(2)}=0
$$

where, as pointed out earlier, we have to substitute the chiral super expansion (46) and (50) into the r.h.s. of (60) and, then only, we have to operate $\partial_{\theta}$. The above equation (61) is nothing but the proof for the off-shell nilpotency $\left[\left(Q_{a b}^{(2)}\right)^{2}=0\right]$ of the anti-BRST charge which becomes transparent when we exploit the beauty of the relationship between the continuous symmetries and their generators as we have shown in Equation (34). Thus, we have captured the off-shell nilpotency of the anti-BRST charge within the framework of ACSA.

At this juncture, we pay our attention to capture the absolute anticommutativity of the BRST charge with the anti-BRST charge using the theoretical power of ACSA to BRST formalism. In this context, we note that the BRST charge $Q_{b}^{(2)}$ (cf. Equation (30)] can be also generalized onto the $(1,1)$-dimensional chiral super submanifold as

$$
\begin{aligned}
Q_{b}^{(2)} \rightarrow & \tilde{Q}_{b}^{(2)}(\tau, \theta) \\
= & B^{(a b)}(\tau, \theta) \dot{F}^{(a b)}(\tau, \theta)-\dot{B}^{(a b)}(\tau, \theta) F^{(a b)}(\tau, \theta) \\
& +2 i E^{(a b)}(\tau, \theta) \tilde{\beta}^{(a b)}(\tau, \theta) \Gamma^{(a b)}(\tau, \theta)+2 \tilde{\beta}^{(a b)} \\
& \times(\tau, \theta) \widetilde{\bar{\beta}}^{(a b)}(\tau, \theta) \dot{F}^{(a b)}(\tau, \theta)-2 F^{(a b)}(\tau, \theta) \\
& \times\left[\widetilde{\bar{\beta}}^{(a b)}(\tau, \theta) \dot{\tilde{\beta}}^{(a b)}(\tau, \theta)+\Gamma^{(a b)}(\tau, \bar{\theta}) \tilde{\chi}^{(a b)}(\tau, \theta)\right] \\
& \left.+2 B^{(a b)}(\tau, \theta) \tilde{\beta}^{(a b)}(\tau, \theta) \tilde{\chi}^{(a b)}(\tau, \theta)-2 i m \Gamma^{(a b)}\right) \\
& \times(\tau, \theta) \tilde{\beta}^{(a b)}(\tau, \theta)-\tilde{\beta}^{(a b)}(\tau, \theta) \tilde{\beta}^{(a b)}(\tau, \theta) \dot{\bar{F}}^{(a b)}(\tau, \theta) \\
& \times+2 \tilde{\chi}^{(a b)}(\tau, \theta) \tilde{\beta}^{(a b)}(\tau, \theta) \tilde{\beta}^{(a b)}(\tau, \theta) \widetilde{\bar{\beta}}^{(a b)}(\tau, \theta),
\end{aligned}
$$

where the chiral supervariables on the r.h.s. are nothing but the super expansions that have been quoted in (46) and (50). It is worthwhile to point out that some of the supervariables are ordinary variables in the true sense of the word (cf. Equation (46)). We can now operate by the Grassmannian derivative $\partial_{\theta}$ on (62) to produce

$$
\begin{aligned}
\frac{\partial}{\partial \theta} \tilde{Q}_{b}^{(2)}(\tau, \theta) \equiv & \int d \theta \tilde{Q}_{b}^{(2)}(\tau, \bar{\theta}) \\
= & i(b+\bar{b}+2 \beta \bar{\beta})[\dot{\bar{b}}+2 \chi \gamma+2 \beta \dot{\bar{\beta}}] \\
& -i \bar{b} \frac{d}{d \tau}[b+\bar{b}+2 \beta \bar{\beta}] \equiv s_{a b} Q_{b}^{(2)} \\
= & -i\left\{Q_{b}^{(2)}, Q_{(a b)}^{(2)}\right\} .
\end{aligned}
$$

In the above, we have utilized the mapping $\partial_{\theta} \Leftrightarrow s_{a b}$ to express the 1.h.s. in the ordinary space. The expression $s_{a b} Q_{b}^{(2)}$ is nothing but the absolute anticommutativity (i.e., $\left.\left\{Q_{b}^{(2)}, Q_{a b}^{(2)}\right\}\right)$ of the BRST charge with the anti-BRST charge $Q_{a b}^{(2)}$. It is crystal clear that the absolute anticommutativity property (i.e., $\left\{Q_{b}^{(2)}, Q_{a b}^{(2)}\right\}=0$ ) is satisfied if and only if we impose the condition $(b+\bar{b}+2 \beta \bar{\beta}=0)$. In other words, we have been able to derive the CF-type restriction: $b+\bar{b}+2 \beta \bar{\beta}=0$ (which characterizes a BRST quantized theory) by exploiting the theoretical tricks and techniques of ASCA to BRST formalism.

Ultimately, we concentrate to capture the absolute anticommutativity of the anti-BRST charge $Q_{a b}^{(2)}$ with the BRST charge $Q_{b}^{(2)}$ within the framework of ACSA to BRST formalism. Toward this goal in mind, we generalize the anti-BRST charge $Q_{a b}^{(2)}$ (cf. Equation (33)) onto the $(1,1)$-dimensional antichiral super submanifold as

$$
\begin{aligned}
Q_{a b}^{(2)} \rightarrow & \tilde{Q}_{a b}^{(2)}(\tau, \bar{\theta}) \\
= & \dot{\bar{B}}^{(b)}(\tau, \bar{\theta}) \bar{F}^{(b)}(\tau, \bar{\theta})-\bar{B}^{(b)}(\tau, \bar{\theta}) \dot{\bar{F}}^{(b)}(\tau, \bar{\theta}) \\
& +2 i E^{(b)}(\tau, \bar{\theta}) \widetilde{\bar{\beta}}^{(b)}(\tau, \bar{\theta}) \Gamma^{(b)}(\tau, \bar{\theta}) \\
& -2 \tilde{\beta}^{(b)}(\tau, \bar{\theta}) \widetilde{\bar{\beta}}^{(b)}(\tau, \bar{\theta}) \dot{\bar{F}}^{(b)}(\tau, \bar{\theta})+2 \bar{F}^{(b)}(\tau, \bar{\theta}) \\
& \times\left[\dot{\overline{\bar{\beta}}}^{(b)}(\tau, \bar{\theta}) \tilde{\beta}^{(b)}(\tau, \bar{\theta})-\Gamma^{(b)}(\tau, \bar{\theta}) \tilde{\chi}^{(b)}(\tau, \bar{\theta})\right] \\
& -2 \bar{B}^{(b)}(\tau, \bar{\theta}) \widetilde{\bar{\beta}}^{(b)}(\tau, \bar{\theta}) \tilde{\chi}^{(b)}(\tau, \bar{\theta})-2 i m \Gamma^{(b)} \\
& \times(\tau, \bar{\theta}) \widetilde{\bar{\beta}}^{(b)}(\tau, \bar{\theta})+\widetilde{\bar{\beta}}^{(b)}(\tau, \bar{\theta}) \widetilde{\bar{\beta}}^{(b)}(\tau, \bar{\theta}) \dot{F}^{(b)}(\tau, \bar{\theta}) \\
& -2 \tilde{\chi}^{(b)}(\tau, \bar{\theta}) \widetilde{\bar{\beta}}^{(b)}(\tau, \bar{\theta}) \widetilde{\bar{\beta}}^{(b)}(\tau, \bar{\theta}) \tilde{\beta}^{(b)}(\tau, \bar{\theta}),
\end{aligned}
$$

where the antichiral supervariable on the r.h.s. are nothing but the super expansions (40) and (44) that have been derived after the applications of the quantum gauge (i.e., BRST) invariant restrictions on the antichiral supervariables. It goes without saying that some of the supervariables on the r.h.s. of (64) are actually ordinary variables (cf. Equation (40)). In view of our understanding that we have $s_{a b} \Leftrightarrow \partial_{\bar{\theta}}$ [10-12], we can operate a derivation w.r.t. the Grassmannian variable $\bar{\theta}$ on the $\tilde{Q}_{a b}^{(2)}(\tau, \bar{\theta})$ as

$$
\begin{aligned}
\frac{\partial}{\partial \bar{\theta}} \tilde{Q}_{a b}^{(2)}(\tau, \bar{\theta}) \equiv & \int d \bar{\theta} \tilde{Q}_{a b}^{(2)}(\tau, \bar{\theta})=0 \\
= & i b \frac{d}{d \tau}[b+\bar{b}+2 \beta \bar{\beta}]-i(b+\bar{b}+2 \beta \bar{\beta}) \\
& \cdot[\dot{b}+2 \beta \bar{\beta}+2 \gamma \chi] \Leftrightarrow s_{b} Q_{a b}^{(2)} \\
= & -i\left\{Q_{a b}^{(2)}, Q_{b}^{(2)}\right\} .
\end{aligned}
$$


In other words (cf. Equation (38)), we have captured the absolute anticommutativity property of the anti-BRST charge with the BRST charge within the framework of ACSA to BRST formalism. A close look at (65) demonstrates that the r.h.s. of $\partial_{\bar{\theta}} \tilde{Q}_{b}^{(2)}(\tau, \bar{\theta})$ is zero if and only if the CF-type restriction is imposed from outside. In other words, we have proven the existence of the CF-type restriction: $b+$ $\bar{b}+2 \beta \bar{\beta}=0$ (on our BRST quantized theory) within the framework of ACSA to BRST formalism.

We end this section with the following remarks. First and foremost, we observe that the mappings: $s_{b} \leftrightarrow \partial_{\bar{\theta}}, s_{a b} \leftrightarrow \partial_{\theta}$ [10-12], play a crucial role in the proof of the two decisive properties of the (anti-)BRST conserved charges. Second, it is interesting to point out that the conserved charges $Q_{(a) b}^{(2)}$ can be generalized onto $(1,1)$-dimensional (anti-)chiral super submanifolds of the general $(1,2)$-dimensional supermanifold. Finally, the operations of the translational generators $\left(\partial_{\theta}, \partial_{\bar{\theta}}\right)$ (along the chiral and antichiral directions of the super submanifolds) on the generalized forms of the supercharges $\tilde{Q}_{(a) b}^{(2)}$ lead to the proof of the off-shell nilpotency (cf. Equations (59) and (61)) and absolute anticommutativity properties of the (anti-)BRST charges as well as the deduction of the (anti-)BRST invariant CF-type restriction (cf. Equations (63) and (65)) within the framework of ACSA to BRST formalism.

\section{Conclusions}

In our present endeavor, we have done a thread-bare analysis of the classical gauge, supergauge, and reparameterization symmetries of the first-order Lagrangian for the 1D system of a massive spinning relativistic particle. We have demonstrated that, in specific limits and identifications, the reparameterization symmetries incorporate the gauge and (super)gauge symmetries (cf. Section 2). We have established that the constraints of our theory (described by the firstorder Lagrangian) are of first-class variety in the terminology of Dirac's prescription for the classification scheme $[36,37]$. We have obtained the secondary constraints from the equivalent Lagrangians and corresponding canonical Hamiltonians of our 1D system of spinning relativistic particle (cf. Section 2 and Appendix A).

We have elevated the combined classical gauge and supergauge symmetry transformations (cf. Equation (7)) to its counterpart quantum (anti-)BRST symmetry transformations which are respected by the coupled (but equivalent) (anti-)BRST invariant Lagrangians. The hallmark of a quantum theory (discussed within the purview of the BRST approach) is the existence of the CF-type restriction(s). We have demonstrated the existence of a single CF-type restriction on our theory by demanding the absolute anticommutativity of the off-shell nilpotent (anti-)BRST symmetry transformations as well as by proving the equivalence of the coupled Lagrangians with respect to the quantum gauge (i.e., (anti-)BRST) symmetry transformations (cf. Equations (22) and (23)). In other words, the absolute anticommutativity of the (anti-)BRST symmetry transformations and the existence of the coupled (but equivalent) Lagrangians owe their origins to the CF-type restriction which defines a submanifold in the quantum Hilbert space of variables that is defined by the equation: $b+\bar{b}+2 \beta \bar{\beta}=0$.

To corroborate the sanctity of our (anti-)BRST symmetry transformations, coupled (but equivalent) Lagrangians, and their invariance(s), we have exploited the theoretical potential and power of ACSA to BRST formalism [20-24] where only the (anti-)chiral supervariables and their suitable expansion(s) along the Grassmannian direction(s) have been considered. One of the novel observations, in this context, has been the proof of absolute anticommutativity of the conserved and nilpotent (anti-)BRST charges within the framework of ACSA to BRST formalism where we have considered only the (anti-)chiral super expansions. This proof, it should be emphasized, is obvious when the full expansions of the supervariables (defined on the full supermanifold) are taken into account. The importance of the ACSA to BRST formalism lies in its simplicity and its dependence on the quantum gauge (i.e., (anti-)BRST) invariant restrictions on the supervariables which are defined on the (anti-)chiral super submanifolds of the general (full) supermanifold. Whereas the (anti-)chiral super submanifolds are characterized by a single Grassmannian variable, the general supermanifold is defined by the superspace coordinates that incorporate a pair of Grassmannian variables. The quantum (anti-)BRST symmetry transformations are found to be associated with the translational generators $\left(\partial_{\theta}, \partial_{\bar{\theta}}\right)$ along the Grassmannian directions $(\theta, \bar{\theta})$.

We have devoted a great of discussion on the derivation and proof of the existence of CF-type restriction (see, also, e.g., [25]) on our theory because the hallmark $[5,6]$ of a quantum theory (described and discussed within the framework of BRST formalism) is its presence. We have shown its appearance in the context of absolute anticommutativity of the (anti-)BRST symmetries (cf. Equation (14)), invariance and equivalence of the coupled (but equivalent) Lagrangians (cf. Equations (23)-(25)), and absolute anticommutativity of the conserved and nilpotent (anti-)BRST charges (cf. Equations (35) and (37)) in the ordinary space. These features have also been captured in the superspace by exploiting the theoretical potential and power of ACSA to BRST formalism (cf. Sections 6 and 7).

We would like to comment on the various kinds of superfield approaches (e.g., USFA, AVSA, and ACSA) to BRST formalism that have been developed over the years. The USFA is the one where mathematically beautiful HC has been exploited to derive the (anti-)BRST symmetry transformations for the gauge and associated (anti-)ghost fields in the case of a (non-)Abelian 1-form gauge theory (see, e.g., [10-12]). In addition, it has led to the systematic derivation of the (anti-)BRST invariant CF condition [7]. The AVSA is a minor extension of the USFA (developed in [10-12]) where the $\mathrm{HC}$ and gauge-invariant restriction(s) play an important role together for the derivation of the proper (anti-)BRST symmetry transformations of the gauge, (anti-)ghost, and matter fields together for an interacting gauge theory. The ACSA is a simplified version of USFA 
where the quantum gauge (i.e., (anti-)BRST) invariant restrictions on the superfields/supervariables lead to the derivation of the (anti-)BRST symmetry transformations for all the fields. Within the framework of ACSA, the (anti-)BRST invariant CF-type restriction(s) arise in the proof of (i) the invariance of the coupled (but equivalent) Lagrangian densities and (ii) the absolute anticommutativity of the conserved and nilpotent (anti-)BRST charges.

In our present investigation, we have performed the BRST and supervariable analysis of a toy model (i.e., 1D system) of a massive spinning relativistic particle where only the (super)gauge symmetry transformations (7) have been exploited for the BRST analysis. We have not devoted any time on the BRST analysis corresponding to the infinitesimal reparameterization symmetries (4). It would be a nice future endeavor to exploit the latter symmetry transformations for the BRST analysis in view of the fact that such an exercise has already been performed by us in the case of a scalar relativistic particle [26]. We plan to extend the richness of our theoretical analysis to the realm of interesting systems of the quantum field theory as well as diffeomorphism invariant theories of gravitation and (super)strings. It is worthwhile to mention here that, in a recent set of papers (see, e.g., [38-40]), the BRST analysis has been performed for the celebrated $\mathrm{ABJM}$ theory. It would be, therefore, a very nice future project for us to apply our present theoretical analysis to the ABJM theory. We are currently very seriously involved with the classical diffeomorphism symmetry and its elevation to the quantum (anti-)BRST symmetries for the system of scalars, vectors, and metric tensor. Our results, in this direction, would be reported elsewhere [41].

\section{Appendix}

\section{A. On the Derivation of Secondary Constraints}

The purpose of our present appendix is to derive the secondary constraints $p^{2}-m^{2} \approx 0$ and $p_{\mu} \psi^{\mu}-m \psi_{5} \approx 0$ from all the three equivalent Lagrangians (1) as well as from the corresponding Hamiltonians (11). First of all, we focus on $L_{0}$ and $H_{c}^{(0)}$. It is evident that the expression for the canonical conjugate momenta $\left(p_{\mu}\right)$ w.r.t. the coordinate $\left(x^{\mu}\right)$ is

$$
p_{\mu}=\frac{\partial L_{0}}{\partial \dot{x}^{\mu}}=\frac{m\left(\dot{x}_{\mu}+i \chi \psi_{\mu}\right)}{\sqrt{\left(\dot{x}^{\rho}+i \chi \psi^{\rho}\right)\left(\dot{x}_{\rho}+i \chi \psi_{\rho}\right)}} .
$$

It is self-evident that the Euler-Lagrange equation of motion (EL-EOM) for our free system is $\dot{p}_{\mu}=d p_{\mu} / d \tau=0$ and it satisfies the mass-shell condition:

$$
p_{\mu} p^{\mu}=\frac{m^{2}\left(\dot{x}_{\mu}+i \chi \psi_{\mu}\right)\left(\dot{x}^{\mu}+i \chi \psi^{\mu}\right)}{\left(\sqrt{\left(\dot{x}^{\rho}+i \chi \psi^{\rho}\right)\left(\dot{x}_{\rho}+i \chi \psi_{\rho}\right)}\right)^{2}}=m^{2} .
$$

Furthermore, it is evident from $L_{0}$ that we have the expression for the canonical conjugate momentum w.r.t. the variable $\chi$ (with $\Pi_{\chi}$ weakly equal to zero) as

$$
\Pi_{\chi}=\frac{\partial L_{0}}{\partial \dot{\chi}}=0 \Rightarrow \Pi_{\chi} \approx 0
$$

Thus, we have the primary constraint as $\Pi_{\chi} \approx 0$ (weekly zero). Hence, we are allowed to take a first-order time derivative on it in the EL-EOM w.r.t. $\chi$ as (see, e.g., $[36,37,42]$ ):

$$
\frac{d}{d \tau}\left(\frac{\partial L_{0}}{\partial \dot{\chi}}\right)=\frac{\partial L_{0}}{\partial \chi} \Rightarrow \dot{\Pi}_{\chi}=+i\left(p_{\mu} \psi^{\mu}-m \psi_{5}\right) \approx 0,
$$

which leads to the derivation of the secondary constraint as $\left(p_{\mu} \psi^{\mu}-m \psi_{5}\right) \approx 0$. The same result is also obtained from the canonical Hamiltonian $H_{c}^{(0)}$ as we note that the Heisenberg EOMs for the time derivative on the conjugate momenta operators $\left(p_{\mu}, \Pi_{\chi}\right)$ are

$$
\begin{aligned}
& \dot{p}_{\mu}=-i\left[p_{\mu}, H_{c}^{(0)}\right]=0, \\
& \dot{\Pi}_{\chi}=-i\left[\Pi_{\chi}, H_{c}^{(0)}\right]=i\left(p_{\mu} \psi^{\mu}-m \psi_{5}\right) \approx 0 .
\end{aligned}
$$

Hence, we have derived the secondary constraints $\left(p^{2}-m^{2}\right) \approx 0$ and $\left(p_{\mu} \psi^{\mu}-m \psi_{5}\right) \approx 0$ from the Lagrangian $L_{0}$ with square root and the corresponding canonical Hamiltonian $H_{c}^{(0)}$ (which, primarily, is nothing but the secondary constraint on our theory).

Now, the stage is set to derive the primary and secondary constraints from the Lagrangians $L_{f}$ and $L_{s}$ (cf. Equation (1)) and corresponding canonical Hamiltonian $H_{c}$ (cf. Equation (11)). It is evident that the expressions for the canonical conjugate momenta w.r.t. the variables $e$ and $\chi$, from $L_{f}$ and $L_{s}$, are

$$
\begin{aligned}
\Pi_{e} & =\frac{\partial L_{r}}{\partial \dot{e}} \approx 0, \\
\Pi_{\chi} & =\frac{\partial L_{r}}{\partial \dot{\chi}} \approx 0, \\
r & =f, s .
\end{aligned}
$$

Hence, we have primary constraints $\Pi_{e} \approx 0$ and $\Pi_{\chi} \approx 0$ (i.e., weekly zero). As per the Dirac prescription (see, e.g., $[36,37,42])$, we are allowed to take a first-order time derivative on these primary constraints. Using the ELEOMs w.r.t. $e$ and $\chi$ variables, we find that

$$
\begin{aligned}
& \frac{d}{d \tau}\left(\frac{\partial L_{f}}{\partial \dot{e}}\right)=\frac{\partial L_{f}}{\partial e} \Rightarrow \dot{\Pi}_{e}=-\frac{1}{2}\left(p^{2}-m^{2}\right) \approx 0, \\
& \frac{d}{d \tau}\left(\frac{\partial L_{f}}{\partial \dot{\chi}}\right)=\frac{\partial L_{f}}{\partial \chi} \Rightarrow \dot{\Pi}_{\chi}=-i\left(p_{\mu} \psi^{\mu}-m \psi_{5}\right) \approx 0 .
\end{aligned}
$$


It is clear, from the above, that we have already derived the secondary constraints $p^{2}-m^{2} \approx 0$ and $p_{\mu} \psi^{\mu}$ $-m \psi_{5} \approx 0$. As far as the Lagrangian $L_{s}$ is concerned, we have the expression for the canonical conjugate momenta $\left(p_{\mu}\right)$ w.r.t. the coordinates $\left(x^{\mu}\right)$ as

$$
p_{\mu}=\frac{\partial L_{s}}{\partial \dot{x}^{\mu}}=\frac{\left(\dot{x}_{\mu}+i e \psi_{\mu}\right)}{e} .
$$

The EL-EOM w.r.t. $e$ (from the second-order Lagrangian $L_{s}$ ) yields

$$
\begin{aligned}
\frac{d}{d \tau}\left(\frac{\partial L_{s}}{\partial \dot{e}}\right) & =\frac{\partial L_{s}}{\partial e} \Rightarrow \dot{\Pi}_{e} \\
& =-\frac{1}{2} \frac{\left(\dot{x}_{\mu}+i \chi \psi_{\mu}\right)\left(\dot{x}^{\mu}+i \chi \psi^{\mu}\right)}{e^{2}}+\frac{m^{2}}{2} \\
& \equiv-\frac{1}{2}\left(p^{2}-m^{2}\right) \approx 0,
\end{aligned}
$$

which produces the secondary constraint $p^{2}-m^{2} \approx 0$. Similarly, the EL-EOM w.r.t. $\chi$ is

$$
\frac{d}{d \tau}\left(\frac{\partial L_{s}}{\partial \dot{\chi}}\right)=\frac{\partial L_{s}}{\partial \chi} \Rightarrow \dot{\Pi}_{\chi}=-i\left(p_{\mu} \psi^{\mu}-m \psi_{5}\right) \approx 0 .
$$

Hence, we have derived both the secondary constraints $p^{2}-m^{2} \approx 0$ and $p_{\mu} \psi^{\mu}-m \psi_{5} \approx 0$ from the first- and secondorder Lagrangians $L_{f}$ and $L_{s}$, respectively.

Against the backdrop of the existence of the primary constraints $\Pi_{e} \approx 0$ and $\Pi_{\chi} \approx 0$, we derive the secondary constraints from the canonical Hamiltonian $H_{c}$ (cf. Equation (11)) as follows (with the natural units $\hbar=c=1$ ), namely,

$$
\begin{aligned}
& \dot{\Pi}_{e}=-i\left[\Pi_{e}, H_{c}\right]=-\frac{1}{2}\left(p^{2}-m^{2}\right) \approx 0, \\
& \dot{\Pi}_{\chi}=-i\left[\Pi_{\chi}, H_{c}\right]=-i\left(p_{\mu} \psi^{\mu}-m \psi_{5}\right) \approx 0,
\end{aligned}
$$

where we have used the canonical commutator $\left[e, \Pi_{e}\right]=i$ and canonical anticommutator as $\left\{\chi, \Pi_{\chi}\right\}=i$ in the natural units $\hbar=c=1$. We end this appendix with the remark that we have derived the secondary constraints $p^{2}-m^{2} \approx 0$ and $p_{\mu} \psi^{\mu}-$ $m \psi_{5} \approx 0$ from all the three equivalent Lagrangian (1) as well as from the canonical Hamiltonian (11). As a passing remark, we note that the whole dynamics of our theory is governed by the secondary constraints because a close look at $H_{c}$ (cf. Equation (11)) demonstrates that the Hamiltonian is a linear combination of the constraints $p^{2}-m^{2} \approx 0$ and $p_{\mu} \psi^{\mu}-m$ $\psi_{5} \approx 0$. Last but not the least, we note that the constraint $p_{\mu}$ $\psi^{\mu}-m \psi_{5} \approx 0$ is the square root of the mass-shell condition $p^{2}-m^{2} \approx 0$ because we observe the following:

$$
\left(p_{\mu} \psi^{\mu}-m \psi_{5}\right)^{2}=\frac{1}{2}\left\{p_{\mu} \psi^{\mu}-m \psi_{5}, p_{\nu} \psi^{\nu}-m \psi_{5}\right\} .
$$

It is straightforward to note, from the first-order and second-order Lagrangians, that we have the following explicit expressions, namely,

$$
\begin{gathered}
\Pi_{(\psi)}^{\mu}=-\frac{i}{2} \psi^{\mu}, \\
\Pi_{\left(\psi_{5}\right)}=\frac{i}{2} \psi_{5},
\end{gathered}
$$

as the canonical conjugate momenta w.r.t. the fermionic variables $\psi_{\mu}$ and $\psi_{5}$. As a consequence, we have the following canonical anticommutators:

$$
\begin{aligned}
& \left\{\psi_{\mu}, \psi_{\nu}\right\}=-2 \eta_{\mu \nu}, \\
& \left\{\psi_{5}, \psi_{5}\right\}=2 \Rightarrow \psi_{5}^{2}=1 .
\end{aligned}
$$

Using the above anticommutators (A.14), we find that the r.h.s. of (A.12) is nothing but the mass-shell condition: $p^{2}-m^{2}=0$. This observation establishes the fact that the two secondary constraints (i.e., $p^{2}-m^{2} \approx 0, p_{\mu} \psi^{\mu}-m \psi_{5}$ $\approx 0$ ) of the theory are interrelated.

\section{B. On the Derivation of Conserved Noether Charges}

The central goal of our present appendix is to derive the (anti-)BRST charges $Q_{(a) b}^{(1)}$ from the basic principle of Noether's theorem and prove their conservation law by exploiting the EL-EOMs that emerge out from the coupled (but equivalent) Lagrangians $L_{b}$ and $L_{\bar{b}}$. First of all, we concentrate on the derivation of the anti-BRST charge $Q_{a b}^{(1)}$ by using the following standard formula for the Noether charge in $1 \mathrm{D}$, namely,

$$
\begin{aligned}
Q_{a b}^{(1)}= & \left(s_{a b} x_{\mu}\right)\left(\frac{\partial L_{\bar{b}}}{\partial \dot{x}_{\mu}}\right)+\left(s_{a b} \psi_{\mu}\right)\left(\frac{\partial L_{\bar{b}}}{\partial \dot{\psi}_{\mu}}\right)+\left(s_{a b} \psi_{5}\right)\left(\frac{\partial L_{\bar{b}}}{\partial \dot{\psi}_{5}}\right) \\
& +\left(s_{a b} e\right)\left(\frac{\partial L_{\bar{b}}}{\partial \dot{e}}\right)+\left(s_{a b} \beta\right)\left(\frac{\partial L_{\bar{b}}}{\partial \dot{\beta}}\right)+\left(s_{a b} c\right)\left(\frac{\partial L_{\bar{b}}}{\partial \dot{c}}\right) \\
& +\left(s_{a b} \bar{c}\right)\left(\frac{\partial L_{\bar{b}}}{\partial \dot{\bar{c}}}\right)-\frac{\bar{c}}{2}\left(p^{2}+m^{2}\right)-\frac{\bar{\beta}}{2}\left(p_{\mu} \psi^{\mu}+m \psi_{5}\right) \\
& +\bar{b}(\dot{\bar{c}}+2 \bar{\beta} \chi),
\end{aligned}
$$

where we have taken into account the transformation property of the Lagrangian (cf. Equation (21)) under the antiBRST symmetry transformation $\left(s_{a b}\right)$ that is quoted in Equation (12). Furthermore, we have utilized our knowledge of the transformations: $s_{a b} \bar{\beta}=0$ and $s_{a b} \gamma=0$. In addition, we have also noted that $\left(\partial L_{\bar{b}} / \partial \dot{\chi}\right)=0$ and $\left(\partial L_{\bar{b}} / \partial \dot{b}\right)=0$. We would like to lay emphasis on the fact that we have taken into account the convention of the left derivative w.r.t. the fermionic variables $\left(\psi_{\mu}, \psi_{5}, c, \bar{c}\right)$. Hence, the expression for the 
anti-BRST charge in Equation (B.1) is correct according to our adopted convention of the derivatives.

The substitutions of the transformations (12) and the proper expressions for the derivatives into (B.1) lead to the exact expression for the anti-BRST charge $\left(Q_{a b}^{(1)}\right)$ that has been quoted in Equation (26). The conservation law (i.e., $\dot{Q}_{a b}^{(1)}=0$ ) can be proven by taking into account the following EL-EOMs that emerge out from the Lagrangian $L_{\bar{b}}$, namely,

$$
\begin{aligned}
& \dot{p}_{\mu}=0, \\
& \dot{x}_{\mu}=e p_{\mu}-i \chi \psi_{\mu}, \\
& \dot{\psi}_{\mu}=\chi p_{\mu}, \\
& 2 \bar{b}-\dot{e}+2 \beta \bar{\beta}=0, \\
& \dot{\psi}_{5}=m \chi-i \dot{\gamma} \equiv 2 e \chi-m \chi-2(\beta \bar{c}-\bar{\beta} c), \\
& \ddot{c}+2 \beta(\dot{\chi}+i \gamma)+2 \dot{\beta} \chi=0, \\
& \ddot{\bar{c}}+2 \bar{\beta}(\dot{\chi}+i \gamma)+2 \chi \dot{\bar{\beta}}=0, \\
&(m-e) \dot{\bar{\beta}}=\bar{\beta}(\bar{b}+\beta \bar{\beta})+i \chi \dot{\bar{c}}+\gamma \bar{c}, \\
&(e-m) \dot{\beta}=\beta(\bar{b}+\beta \bar{\beta}-\dot{e})-i \chi \dot{c}+\gamma c .
\end{aligned}
$$

It would be noted that we have not incorporated, in the above, the EL-EOMs w.r.t. the variables $e$ and $\chi$ because these have already been invoked and utilized in Equation (32).

At this stage, we focus on the derivation of the Noether conserved charge $Q_{b}^{(1)}$ (cf. Equation (27)). Applying the basic concept behind the Noether theorem, we note that we have the following expression for the BRST charge, namely,

$$
\begin{aligned}
Q_{b}^{(1)}= & \left(s_{b} x_{\mu}\right)\left(\frac{\partial L_{b}}{\partial \dot{x}_{\mu}}\right)+\left(s_{b} \psi_{\mu}\right)\left(\frac{\partial L_{b}}{\partial \dot{\psi}_{\mu}}\right)+\left(s_{b} \psi_{5}\right)\left(\frac{\partial L_{b}}{\partial \dot{\psi}_{5}}\right) \\
& +\left(s_{b} e\right)\left(\frac{\partial L_{b}}{\partial \dot{e}}\right)+\left(s_{b} \bar{\beta}\right)\left(\frac{\partial L_{b}}{\partial \dot{\bar{\beta}}}\right)+\left(s_{b} c\right)\left(\frac{\partial L_{b}}{\partial \dot{c}}\right) \\
& +\left(s_{b} \bar{c}\right)\left(\frac{\partial L_{b}}{\partial \dot{\bar{c}}}\right)-\frac{c}{2}\left(p^{2}+m^{2}\right)-\frac{\beta}{2}\left(p_{\mu} \psi^{\mu}+m \psi_{5}\right) \\
& -b(\dot{c}+2 \beta \chi),
\end{aligned}
$$

where we have taken into account the trivial transformations $s_{b} \beta=0, s_{b} \gamma=0$ as well as the fact that $\left(\partial L_{b} / \partial \dot{\chi}\right)=0$ and $\left(\partial L_{b} / \partial \dot{\bar{b}}\right)=0$. The substitutions of the transformations (13) and the proper and precise values of the derivatives (derived from $L_{b}$ ) lead to the derivation of the conserved charge $Q_{b}^{(1)}$ that has been listed in Equation (27). The conservation law $\dot{Q}_{b}^{(1)}=0$ can be proven by taking into account the following EL-EOMs that emerge out from the Lagrangian $L_{b}$, namely,

$$
\begin{gathered}
\dot{p}_{\mu}=0, \\
\dot{x}_{\mu}=e p_{\mu}-i \chi \psi_{\mu}, \\
\dot{\psi}_{\mu}=\chi p_{\mu}, \\
2 b+\dot{e}+2 \beta \bar{\beta}=0, \\
\dot{\psi}_{5}=2 e \chi-2(\beta \bar{c}-\bar{\beta} c)-m \chi \equiv m \chi-i \dot{\gamma}, \\
\ddot{\bar{c}}+2 \bar{\beta}(\dot{\chi}+i \gamma)+2 \chi \dot{\bar{\beta}}=0, \\
\ddot{c}+2 \beta(\dot{\chi}-i \gamma)+2 \dot{\beta} \chi, \\
(m-e) \dot{\beta}+b \beta+\bar{\beta} \beta^{2}-i \chi \dot{c}-\gamma c=0, \\
(m-e) \dot{\bar{\beta}}-(b+\dot{e}) \bar{\beta}-\bar{\beta}^{2} \beta-i \chi \dot{\bar{c}}-\gamma \bar{c}=0 .
\end{gathered}
$$

We would like to point out that, in the above, we have not incorporated a couple of equations (hidden in Equation (29)) which are the EL-EOMs w.r.t. the variables $e$ and $\chi$ from $L_{b}$.

We end this appendix with the remarks that we have derived the Noether conserved (anti-)BRST charges $Q_{(a) b}^{(1)}$ (cf. Equations (26) and (27)) which are only on-shell nilpotent of order two. To accomplish the off-shell nilpotency (without any use of EL-EOMs and/or CF-type restriction), it is essential for us to use the EL-EOMs w.r.t. $e$ and $\chi$ (cf. Equations (29) and (32)) to convert the Noether conserved (anti-)BRST charges $\left(Q_{(a) b}^{(1)}\right)$ into $Q_{(a) b}^{(2)}$ (cf. Equations (30) and (33)).

\section{On the Step-by-Step Derivation of the BRST Symmetry Transformations by Using the ACSA to BRST Formalism}

In our present appendix, we derive the BRST symmetry transformations (13) by exploiting the theoretical potential and power of ACSA to BRST formalism in a systematic fashion. In other words, we obtain the relationships in (43) from the BRST invariant restrictions on the antichiral supervariables in (42). In this context, we note that

$$
\begin{aligned}
\widetilde{\bar{\beta}}(\tau, \bar{\theta}) \Gamma^{(b)}(\tau, \bar{\theta}) & =\bar{\beta}(\tau) \gamma(\tau) \Rightarrow\left[\bar{\beta}(\tau)+\bar{\theta} f_{3}(\tau)\right] \gamma(\tau) \\
& =\bar{\beta}(\tau) \gamma(\tau),
\end{aligned}
$$

implies that $f_{3}(\tau) \propto \gamma(\tau)$. In other words, we obtain $f_{3}(\tau)=$ $k \gamma(\tau)$ where $k$ is a numerical constant. Now, we focus on the following restriction on the combination of the antichiral supervariables, namely,

$B^{(b)}(\tau, \bar{\theta}) \widetilde{\bar{\beta}}(\tau, \bar{\theta})+\Gamma^{(b)}(\tau, \bar{\theta}) \bar{F}(\tau, \bar{\theta})=b(\tau) \bar{\beta}(\tau)+\gamma(\tau) \bar{c}(\tau)$

which is precisely the generalization of the BRST invariant quantity: $s_{b}(b \bar{\beta}+\gamma \bar{c})=0$. The substitution of the expansion 
for $\widetilde{\bar{\beta}}(\tau, \bar{\theta})=\bar{\beta}(\tau)+\bar{\theta}[k \gamma(\tau)]$ into the above and use of Equation (40) yield the following relationship:

$$
\begin{aligned}
b(\tau) & {[\bar{\beta}(\tau)+\bar{\theta}(k \gamma)]+\gamma(\tau)\left[\bar{c}(\tau)+\bar{\theta}\left(b_{2}(\tau)\right)\right] } \\
= & b(\tau) \bar{\beta}(\tau)+\gamma(\tau) \bar{c}(\tau) .
\end{aligned}
$$

It is clear, from the above, that we obtain the following:

$$
k b(\tau) \gamma(\tau)-\gamma b_{2}(\tau)=0 \Rightarrow\left[k b(\tau)-b_{2}(\tau)\right] \gamma(\tau)=0
$$

From this relationship, it is clear that if we wish to have $s_{b} \bar{c}=i b$ in our theory, the constant $k$ would turn out to be $k$ $=i$. It would be recalled that $s_{b} \bar{c}=i b$ is a standard transformation w.r.t. the BRST symmetry $\left(s_{b}\right)$ within the framework of BRST formalism. Thus, ultimately, we have derived the following super expansions in terms of (13), namely,

$$
\begin{aligned}
& \bar{F}^{(b)}(\tau, \bar{\theta})=\bar{c}(\tau)+\bar{\theta}(i b(\tau)) \equiv \bar{c}(\tau)+\bar{\theta}\left(s_{b} \bar{c}(\tau)\right), \\
& \widetilde{\bar{\beta}}^{(b)}(\tau, \bar{\theta})=\bar{\beta}(\tau)+\bar{\theta}(i \gamma(\tau)) \equiv \bar{\beta}(\tau)+\bar{\theta}\left(s_{b} \bar{\beta}(\tau)\right) .
\end{aligned}
$$

Thus, we note that coefficients of $\bar{\theta}$ are nothing but the BRST symmetry transformations: $s_{b} c=i b, s_{b} \bar{\beta}=i \gamma$. Now, we concentrate on the BRST invariant quantity: $s_{b}\left(\beta^{2} \bar{\beta}+\right.$ $c \gamma)=0$. This can be generalized onto the $(1,1)$-dimensional antichiral super submanifold as

$$
\begin{aligned}
& \tilde{\beta}^{2(b)}(\tau, \bar{\theta}) \widetilde{\bar{\beta}}^{(b)}(\tau, \bar{\theta})+F(\tau, \bar{\theta}) \Gamma^{b}(\tau, \bar{\theta}) \\
& \equiv \beta^{2}(\tau) \bar{\beta}(\tau)+c(\tau) \gamma(\tau) .
\end{aligned}
$$

The substitutions of $\tilde{\beta}^{(b)}(\tau, \bar{\theta})=\beta(\tau), \quad \Gamma^{b}(\tau, \bar{\theta})=\gamma(\tau)$, $F(\tau, \bar{\theta})=c(\tau)+\bar{\theta} b_{1}(\tau)$, and (C.5) lead to the following explicit expressions for the l.h.s. and r.h.s., namely,

$$
\begin{aligned}
& \beta^{2}(\tau)[\bar{\beta}(\tau)+\bar{\theta}(i \gamma(\tau))]+\left[c(\tau)+\bar{\theta} b_{1}(\tau)\right] \gamma(\tau) \\
& \quad=\beta^{2}(\tau) \bar{\beta}(\tau)+c(\tau) \gamma(\tau) .
\end{aligned}
$$

The straightforward algebra yields: $b_{1}(\tau)=-i \beta^{2}(\tau)$. Hence, we have the following super expansion for the antichiral supervariable $F(\tau, \bar{\theta})$, namely,

$$
F^{(b)}(\tau, \bar{\theta})=c(\tau)+\bar{\theta}\left(-i \beta^{2}(\tau)\right)=c(\tau)+\bar{\theta}\left(s_{b} c(\tau)\right)
$$

We note that the coefficient of $\bar{\theta}$ is nothing but $s_{b} c=$ $-i \beta^{2}$. It is now straightforward to note that, from the BRST invariant quantities: $s_{b}(\dot{c}+2 \beta \chi)=0$ and $s_{b}(\bar{b}+2 \beta \bar{\beta})=0$ and their generalizations onto the $(1,1)$-dimensional antichiral super submanifold (cf. Equation (42)) (with the inputs from (C.5) and (C.8)) lead to the following:

$$
\begin{aligned}
f_{1}(\tau) & =\dot{c}+2 \beta \chi \\
f_{5} & =-2 i \beta \gamma .
\end{aligned}
$$

The above values of the secondary variables yield the following super expansions:

$$
E^{(b)}(\tau, \bar{\theta})=e(\tau)+\bar{\theta}[\dot{c}(\tau)+2 \beta(\tau) \chi(\tau)] \equiv e(\tau)+\bar{\theta}\left(s_{b} e(\tau)\right)
$$

$$
\bar{B}^{(b)}(\tau, \bar{\theta}) \equiv \bar{b}(\tau)+\bar{\theta}(-2 i \beta(\tau) \gamma(\tau)) \equiv \bar{b}(\tau)+\bar{\theta}\left(s_{b} \bar{b}(\tau)\right)
$$

Hence, we have derived the BRST symmetry transformations: $s_{b} e=\dot{c}+2 \beta \chi, s_{b} \bar{b}=-2 i \beta \gamma$, as the coefficients of $\bar{\theta}$. Now, the inputs from Equations (C.5), (C.8), and (C.11) lead to the determination of all the secondary variables (cf. Equation (43)) from the BRST invariant restriction in (42). The superscript $(b)$ on all the supervariables denotes that, in the super expansions of these supervariables, the coefficients of $\bar{\theta}$ are nothing but the off-shell nilpotent BRST symmetry transformations (13) for our theory.

We end this appendix with the following remarks. First, the BRST and anti-BRST invariant restrictions on the (anti-) chiral supervariables (cf. Equations (40), (42), (46), and (48)) are, precisely speaking, the quantum gauge-invariant restrictions which lead to provide the correct relationships among the secondary variables and basic as well as auxiliary variables of the (anti-)BRST invariant theory. Second, the determination of the secondary variables (that lead to the derivation of the anti-BRST symmetry transformations (12) as the coefficients of $\theta$ in the expansion (50)) has been carried out following exactly similar kind of procedure as we have done to derive the relationship (43) from (42) in the case of determination of the BRST symmetry transformations (13) as the coefficient of $\bar{\theta}$ in the expansions (44). Finally, the ACSA to BRST formalism is a simple but beautiful symmetry-based theoretical technique which is applicable to all kinds of physical systems of theoretical interest.

\section{On an Alternative Proof of the Absolute Anticommutativity and the Existence of CF- Type Restriction}

The purpose of our present appendix is to provide an alternative proof of the absolute anticommutativity of the conserved and nilpotent (anti-)BRST charges and the existence of the (anti-)BRST invariant (i.e., $s_{(a) b}(b+\bar{b}+2 \beta \bar{\beta})=0$ ] CF-type restriction: $b+\bar{b}+2 \beta \bar{\beta}=0$, in the ordinary space as well as in the superspace by exploiting the theoretical potential and power of ACSA to BRST formalism. In this context, we note that the off-shell nilpotent $\left(\left[Q_{(a) b}^{(2)}\right]^{2}=0\right)$ (anti-)BRST charges $Q_{(a) b}^{(2)}$ (cf. Equations (33) and (30)) can be expressed as 
follows:

$$
Q_{a b}^{(2)}=s_{a b}\left[i(\bar{c} \dot{c}-\dot{\bar{c}} c)+2 i(\beta \bar{c}+\bar{\beta} c) \chi-2 e \bar{\beta} \beta+2 \gamma \psi_{5}\right],
$$

$Q_{b}^{(2)}=s_{b}\left[i(\dot{\bar{c}} c-\bar{c} \dot{c})-2 i(\bar{\beta} c+\beta \bar{c}) \chi+2 e \bar{\beta} \beta+2 \gamma \psi_{5}\right]$,

where the (anti-)BRST symmetry transformations have been quoted in Equations (13) and (12), respectively. In other words, it is obvious that the BRST charge $Q_{b}^{(2)}$ can be written as a BRST exact quantity and the anti-BRST charge $Q_{a b}^{(2)}$ can be reexpressed in the exact form w.r.t. the anti-BRST symmetry transformation $s_{a b}$ (cf. Equation (12)). Thus, it is transparent now that we have the following explicit relationships, namely,

$$
\begin{aligned}
& s_{a b} Q_{a b}^{(2)}=-i\left\{Q_{a b}^{(2)}, Q_{a b}^{(2)}\right\}=0 \Leftrightarrow\left[Q_{a b}^{(2)}\right]^{2}=0 \Leftrightarrow s_{a b}^{2}=0, \\
& s_{b} Q_{b}^{(2)}=-i\left\{Q_{b}^{(2)}, Q_{b}^{(2)}\right\}=0 \Leftrightarrow\left[Q_{b}^{(2)}\right]^{2}=0 \Leftrightarrow s_{b}^{2}=0,
\end{aligned}
$$

where we have used the fundamental relationship between the continuous symmetry transformations $s_{(a) b}$ and their generators $Q_{(a) b}^{(2)}$. It is evident, from equations (D.3) and (D.4), that the off-shell nilpotency of the (anti-)BRST charges $Q_{(a) b}^{(2)}$ is deeply and intimately connected with the off-shell nilpotency of the (anti-)BRST symmetry transformations $s_{(a) b}$ in the ordinary space. It is worthwhile to point out that these conclusions can not be drawn from our earlier proof of the off-shell nilpotency (cf. Equations (38) and (36)).

In view of the mappings: $s_{b} \rightarrow \partial_{\bar{\theta}}, s_{a b} \rightarrow \partial_{\theta}$, it is clear that the expressions in (D.1) and (D.2) can be expressed in terms of the (anti-)chiral supervariables and the derivatives as well as the differentials w.r.t. the Grassmannian variables as

$$
\begin{aligned}
Q_{a b}^{(2)}= & \frac{\partial}{\partial \theta}\left[i\left(\bar{F}^{(a b)}(\tau, \theta) \dot{F}^{(a b)}(\tau, \theta)-\dot{\bar{F}}^{(a b)}(\tau, \theta) F^{(a b)}(\tau, \theta)\right)\right. \\
& +2 i\left(\tilde{\beta}^{(a b)}(\tau, \theta) \bar{F}^{(a b)}(\tau, \theta)+\widetilde{\bar{\beta}}^{(a b)}(\tau, \theta) F^{(a b)}(\tau, \theta)\right) \\
& \cdot \tilde{\chi}^{(a b)}(\tau, \theta)-2 E^{(a b)}(\tau, \theta) \widetilde{\bar{\beta}}^{(a b)}(\tau, \theta) \tilde{\beta}^{(a b)}(\tau, \theta) \\
& \left.+2 \Gamma^{(a b)}(\tau, \theta) \Psi_{5}^{(a b)}(\tau, \theta)\right] \\
\equiv & \int d \theta\left[i\left(\bar{F}^{(a b)}(\tau, \theta) \dot{F}^{(a b)}(\tau, \theta)-\dot{\bar{F}}^{(a b)}(\tau, \theta) F^{(a b)}(\tau, \theta)\right)\right. \\
& +2 i\left(\tilde{\beta}^{(a b)}(\tau, \theta) \bar{F}^{(a b)}(\tau, \theta)+\widetilde{\bar{\beta}}^{(a b)}(\tau, \theta) F^{(a b)}(\tau, \theta)\right) \\
& \cdot \tilde{\chi}^{(a b)}(\tau, \theta)-2 E^{(a b)}(\tau, \theta) \widetilde{\bar{\beta}}^{(a b)}(\tau, \theta) \tilde{\beta}^{(a b)}(\tau, \theta) \\
& \left.+2 \Gamma^{(a b)}(\tau, \theta) \Psi_{5}^{(a b)}(\tau, \theta)\right],
\end{aligned}
$$

$$
\begin{aligned}
Q_{b}^{(2)}= & \frac{\partial}{\partial \bar{\theta}}\left[i\left(\dot{\bar{F}}^{(b)}(\tau, \bar{\theta}) F^{(b)}(\tau, \bar{\theta})-\bar{F}^{(b)}(\tau, \bar{\theta}) \dot{F}^{(b)}(\tau, \bar{\theta})\right)\right. \\
& -2 i\left(\widetilde{\bar{\beta}}^{(b)}(\tau, \bar{\theta}) F^{(b)}(\tau, \bar{\theta})-\tilde{\beta}^{(b)}(\tau, \bar{\theta}) \bar{F}^{(b)}(\tau, \bar{\theta})\right) \\
& \cdot \tilde{\chi}^{(b)}(\tau, \bar{\theta})+2 E^{(b)}(\tau, \bar{\theta}) \widetilde{\bar{\beta}}^{(b)}(\tau, \bar{\theta}) \tilde{\beta}^{(b)}(\tau, \bar{\theta}) \\
& \left.+2 \Gamma^{(b)}(\tau, \bar{\theta}) \Psi_{5}^{(b)}(\tau, \bar{\theta})\right] \\
\equiv & \int d \bar{\theta}\left[i\left(\dot{\bar{F}}^{(b)}(\tau, \bar{\theta}) F^{(b)}(\tau, \bar{\theta})-\bar{F}^{(b)}(\tau, \bar{\theta}) \dot{F}^{(b)}(\tau, \bar{\theta})\right)\right. \\
& -2 i\left(\widetilde{\bar{\beta}}^{(b)}(\tau, \bar{\theta}) F^{(b)}(\tau, \bar{\theta})-\tilde{\beta}^{(b)}(\tau, \bar{\theta}) \bar{F}^{(b)}(\tau, \bar{\theta})\right) \\
& \cdot \tilde{\chi}^{(b)}(\tau, \bar{\theta})+2 E^{(b)}(\tau, \bar{\theta}) \widetilde{\bar{\beta}}^{(b)}(\tau, \bar{\theta}) \tilde{\beta}^{(b)}(\tau, \bar{\theta}) \\
& \left.+2 \Gamma^{(b)}(\tau, \bar{\theta}) \Psi_{5}^{(b)}(\tau, \bar{\theta})\right] .
\end{aligned}
$$

It is crystal clear that, a close look at the above equations (D.5) and (D.6), leads to the following relationship within the framework of ACSA to BRST formalism:

$$
\begin{aligned}
& \partial_{\theta} Q_{a b}^{(2)}=0 \Leftrightarrow \partial_{\theta}^{2}=0 \Leftrightarrow s_{a b}^{2}=0, \\
& \partial_{\bar{\theta}} Q_{b}^{(2)}=0 \Leftrightarrow \partial_{\bar{\theta}}^{2}=0 \Leftrightarrow s_{b}^{2}=0 .
\end{aligned}
$$

Thus, we have captured the off-shell nilpotency $\left[\left(Q_{(a) b}^{(2)}\right)^{2}\right.$ $=0]$ of the (anti-)BRST charges (cf. Equations (D.3) and (D.4)) within the framework of ACSA and established that the off-shell nilpotency of the (anti-)BRST symmetries and corresponding conserved charges is intimately connected with the nilpotency $\left(\partial_{\bar{\theta}}^{2}=\partial_{\theta}^{2}=0\right)$ of the translational generators $\left(\partial_{\bar{\theta}}, \partial_{\theta}\right)$ along the Grassmannian $(\bar{\theta}) \theta$-directions of the $(1,1)$-dimensional (anti-)chiral super submanifolds (of the general $(1,2)$-dimensional supermanifold). The relationships in (D.3) and (D.4) are sacrosanct, and these are expected results within the framework of ACSA.

We now focus in the derivation of the CF-type restriction $(b+\bar{b}+2 \beta \bar{\beta}=0)$ in the ordinary space as well as in the superspace (by exploiting the basic techniques of ACSA). Toward this goal in mind, first of all, we assume the validity of the CF-type restriction on our theory, right from the beginning. As a consequence, we observe that the (anti-) BRST charges $Q_{(a) b}^{(2)}$ can be expressed in different forms as

$$
\begin{aligned}
Q_{a b}^{(3)}= & b \dot{\bar{c}}-\dot{b} \bar{c}-2 \bar{\beta} \dot{\beta} \bar{c}-2 \bar{c} \gamma \chi+\bar{\beta}^{2} \dot{c}-2 i \bar{\beta}(m-e) \gamma \\
& +2 b \bar{\beta} \chi+2 \bar{\beta}^{2} \beta \chi \\
Q_{b}^{(3)}= & \dot{\bar{b}} c-\bar{b} \dot{c}+2 \dot{\bar{\beta}} \beta c-2 c \gamma \chi-\beta^{2} \dot{\bar{c}}-2 i \beta(m-e) \gamma \\
& -2 \bar{b} \beta \chi-2 \bar{\beta} \beta^{2} \chi .
\end{aligned}
$$


The above forms are very interesting because now we can express the BRST charge as an exact expression w.r.t. the anti-BRST symmetry transformations (12) and the antiBRST charge as an exact quantity w.r.t. the BRST symmetry transformation listed in (13). In other words, we have the following explicit expressions:

$$
\begin{aligned}
& Q_{a b}^{(3)}=s_{b}\left[i \dot{\bar{c}} \bar{c}-2 i \bar{\beta} \bar{c} \chi+e \bar{\beta}^{2}-m \bar{\beta}^{2}\right], \\
& Q_{b}^{(3)}=s_{a b}\left[i c \dot{c}+2 i \beta c \chi-e \beta^{2}+m \beta^{2}\right] .
\end{aligned}
$$

It is straightforward, at this juncture, to note that we have the following:

$$
\begin{gathered}
s_{b} Q_{a b}^{(3)}=-i\left\{Q_{a b}^{(3)}, Q_{b}^{(3)}\right\}=0 \Leftrightarrow s_{b}^{2}=0, \\
s_{a b} Q_{b}^{(3)}=-i\left\{Q_{b}^{(3)}, Q_{a b}^{(3)}\right\}=0 \Leftrightarrow s_{a b}^{2}=0 .
\end{gathered}
$$

The above observations demonstrate that the absolute anticommutativity of the anti-BRST charge with BRST charge is deeply connected with the off-shell nilpotency $\left(s_{b}^{2}=0\right)$ of the BRST symmetry transformations (13). On the other hand, the absolute anticommutativity of the conserved BRST charge with the anti-BRST charge is intimately connected with the off-shell nilpotency $\left(s_{a b}^{2}=0\right)$ of the antiBRST symmetry transformations (12). Thus, even in the ordinary space, a close look at (D.12) and (D.13) demonstrates that the proof of absolute anticommutativity property distinguishes between the off-shell nilpotency of the BRST and anti-BRST symmetry transformations. This exercise, in a subtle manner, also proves the existence of the anti-BRST invariant $\left[s_{(a) b}(b+\bar{b}+2 \beta \bar{\beta})=0\right]$ CF-type restriction: $b+\bar{b}$ $+2 \beta \bar{\beta}=0$, on our theory of a $1 \mathrm{D}$ system of the reparameterization and (super)gauge invariant massive spinning relativistic particle. In this connection, it is worthwhile to point out that the specific forms (cf. Equations (D.8) and (D.9)) of the (anti-)BRST charges have been obtained from the other expressions for the (anti-)BRST charges (cf. Equations (33) and (30)) only after the substitution of the CF-type restriction: $b+\bar{b}+2 \beta \bar{\beta}=0$, of our theory.

We are now in the position to capture the above absolute anticommutativity property within the framework of ACSA. Keeping in our mind the mappings: $s_{b} \leftrightarrow \partial_{\bar{\theta}}, s_{a b} \leftrightarrow \partial_{\theta}$ (see, e.g., Refs. [10-12] for details), it is evident that the observations in (D.10) and (D.11) can be translated into superspace (with the help of ACSA to BRST formalism) as

$$
\begin{aligned}
Q_{a b}^{(3)}= & \frac{\partial}{\partial \bar{\theta}}\left[i \dot{\bar{F}}^{(b)}(\tau, \bar{\theta}) \bar{F}^{(b)}(\tau, \bar{\theta})-2 i \widetilde{\bar{\beta}}^{(b)}(\tau, \bar{\theta}) \bar{F}^{(b)}(\tau, \bar{\theta})\right. \\
& \left.\cdot \chi^{(b)}(\tau, \bar{\theta})+E^{(b)}(\tau, \bar{\theta}) \widetilde{\bar{\beta}}^{2(b)}(\tau, \bar{\theta})-m \widetilde{\bar{\beta}}^{2(b)}(\tau, \bar{\theta})\right] \\
\equiv & \int d \bar{\theta}\left[i \dot{\bar{F}}^{(b)}(\tau, \bar{\theta}) \bar{F}^{(b)}(\tau, \bar{\theta})-2 i \widetilde{\bar{\beta}}^{(b)}(\tau, \bar{\theta}) \bar{F}^{(b)}(\tau, \bar{\theta})\right. \\
& \left.\cdot \chi^{(b)}(\tau, \bar{\theta})+E^{(b)}(\tau, \bar{\theta}) \widetilde{\bar{\beta}}^{2(b)}(\tau, \bar{\theta})-m \widetilde{\bar{\beta}}^{2(b)}(\tau, \bar{\theta})\right],
\end{aligned}
$$

$$
\begin{aligned}
Q_{b}^{(3)}= & \frac{\partial}{\partial \theta}\left[i F^{(a b)}(\tau, \theta) \dot{F}^{(a b)}(\tau, \theta)+2 i \tilde{\beta}^{(a b)}(\tau, \theta) F^{(a b)}(\tau, \theta)\right. \\
& \left.\cdot \chi^{(b)}(\tau, \theta)-E^{(a b)}(\tau, \theta) \tilde{\beta}^{2(a b)}(\tau, \theta)+m \tilde{\beta}^{2(a b)}(\tau, \theta)\right] \\
\equiv & \int d \theta\left[i F^{(a b)}(\tau, \theta) \dot{F}^{(a b)}(\tau, \theta)+2 i \tilde{\beta}^{(a b)}(\tau, \theta) F^{(a b)}(\tau, \theta)\right. \\
& \left.\cdot \chi^{(b)}(\tau, \theta)-E^{(a b)}(\tau, \theta) \tilde{\beta}^{2(a b)}(\tau, \theta)+m \tilde{\beta}^{2(a b)}(\tau, \theta)\right]
\end{aligned}
$$

where the superscripts $(b)$ and $(a b)$ on the (anti-)chiral supervariables have already been explained (cf. Equations (40), (44), (46), and (50)) earlier (see Sections 4 and 5). At this stage, we observe, in a straightforward fashion, that

$$
\begin{aligned}
& \partial_{\bar{\theta}} Q_{a b}^{(3)}=0 \Leftrightarrow \partial_{\bar{\theta}}^{2}=0 \Leftrightarrow s_{b}^{2}=0, \\
& \partial_{\theta} Q_{b}^{(3)}=0 \Leftrightarrow \partial_{\theta}^{2}=0 \Leftrightarrow s_{a b}^{2}=0 .
\end{aligned}
$$

The above equations are the reflections of our earlier observations in (D.12) and (D.13) which are nothing but the proof of absolute anticommutativity property of the conserved and nilpotent (anti-)BRST charges. In other words, we have been able to capture the absolute anticommutativity property within the purview of ACSA to BRST formalism.

We end this appendix with the closing and key remarks that the proof of the absolute anticommutativity property of the (anti-)BRST charges within the framework of ASCA to BRST formalism distinguishes (cf. Equations (D.15) and (D.16)) between the $(1,1)$-dimensional chiral and antichiral super submanifolds of the general $(1,2)$-dimensional supermanifold on which our 1D system of a massive spinning relativistic particle has been generalized. This is due to the fact that the nilpotent $\left(\partial_{\bar{\theta}}^{2}=\partial_{\theta}^{2}=0\right)$ translational generators $\left(\partial_{\bar{\theta}}, \partial_{\theta}\right)$ are defined separately and independently along the (anti-)chiral Grassmannian directions of the (1, 1)-dimensional super submanifolds (of the $(1,2)$-dimensional supermanifold).

\section{Data Availability}

No data were used to support this study.

\section{Conflicts of Interest}

The authors declare that there are no conflicts of interest.

\section{Acknowledgments}

The present investigation has been carried out under the financial supports from BHU-fellowships to A. Tripathi and A. K. Rao from Banaras Hindu University (BHU), Varanasi. B. Chauhan acknowledges the financial support from DST, Government of India, under its INSPIRE-fellowship program. These authors express their deep sense of gratitude to the above local and national funding agencies for their financial supports. 


\section{References}

[1] C. Becchi, A. Rouet, and R. Stora, "The abelian Higgs Kibble model, unitarity of the S-operator," Physics Letters B, vol. 52, no. 3, pp. 344-346, 1974.

[2] C. Becchi, A. Rouet, and R. Stora, "Renormalization of the abelian Higgs-Kibble model," Communications in Mathematical Physics, vol. 42, no. 2, pp. 127-162, 1975.

[3] C. Becchi, A. Rouet, and R. Stora, "Renormalization of gauge theories," Annals of Physics, vol. 98, no. 2, pp. 287-321, 1976.

[4] I. V. Tyutin, "Gauge invariance in field theory and statistical physics in operator formalism," https://arxiv.org/abs/0812 .0580 .

[5] L. Bonora and R. P. Malik, "BRST, anti-BRST and gerbes," Physics Letters B, vol. 655, no. 1-2, pp. 75-79, 2007.

[6] L. Bonora and R. P. Malik, "BRST, anti-BRST and their geometry," Journal of Physics A: Mathematical and Theoretical, vol. 43, no. 37, p. 375403, 2010.

[7] G. Curci and R. Ferrari, "Slavnov transformations and supersummetry," Physics Letters B, vol. 63, no. 1, pp. 91-94, 1976.

[8] J. Thierry-Mieg, "Geometrical reinterpretation of FaddeevPopov ghost particles and BRS transformations," Journal of Mathematical Physics, vol. 21, no. 12, pp. 2834-2838, 1980.

[9] M. Quirós, F. J. de Urries, J. Hoyos, M. L. Mazón, and E. Rodriguez, "Geometrical structure of Faddeev-Popov fields and invariance properties of gauge theories," Journal of Mathematical Physics, vol. 22, no. 8, pp. 1767-1774, 1981.

[10] L. Bonora and M. Tonin, "Superfield formulation of extended BRS symmetry," Physics Letters B, vol. 98, no. 1-2, pp. 48-50, 1981.

[11] L. Bonora, P. Pasti, and M. Tonin, "Extended BRS symmetry in non-Abelian gauge theories," Il Nuovo Cimento A, vol. 64, no. 3, pp. 307-331, 1981.

[12] L. Bonora, P. Pasti, and M. Tonin, "Superspace approach to quantum gauge theories," Annals of Physics, vol. 144, no. 1, pp. 15-33, 1982.

[13] R. Delbourgo, "Extended BRS invariance and OSp (4/2) supersymmetry," Journal of Physics A: Mathematical and General, vol. 15, no. 2, pp. 611-625, 1982.

[14] L. Baulieu and J. Thierry-Mieg, "The principle of BRS symmetry: an alternative approach to Yang-Mills theories," Nuclear Physics B, vol. 197, no. 3, pp. 477-508, 1982.

[15] L. Alvarez-Gaumé and L. Baulieu, "The two quantum symmetries associated with a classical symmetry," Nuclear Physics B, vol. 212, no. 2, pp. 255-267, 1983.

[16] R. P. Malik, "Abelian 2-form gauge theory: superfield formalism," European Physical Journal C: Particles and Fields, vol. 60, no. 3, pp. 457-470, 2009.

[17] R. P. Malik, "Superfield approach to nilpotent symmetries for QED from a single restriction: an alternative to the horizontality condition," Journal of Physics A: Mathematical and Theoretical, vol. 39, no. 33, pp. 10575-10587, 2006.

[18] R. P. Malik, "An alternative to the horizontality condition in the superfield approach to BRST symmetries," European Physical Journal C: Particles and Fields, vol. 51, no. 1, pp. 169-177, 2007.

[19] R. P. Malik, "Superfield approach to symmetries for matter fields in Abelian gauge theories," Journal of Physics A: Mathematical and Theoretical, vol. 37, no. 19, pp. 5261-5274, 2004.

[20] S. Kumar, B. Chauhan, and R. P. Malik, "(Anti-)chiral supervariable approach to nilpotent and absolutely anticommuting conserved charges of reparametrization invariant theories: a couple of relativistic toy models as examples," International Journal of Modern Physics A: Particles and Fields; Gravitation; Cosmology; Nuclear Physics, vol. 33, no. 22, p. 1850133, 2018.

[21] A. Shukla, N. Srinivas, and R. P. Malik, "Nilpotent symmetries of a 4Dmodel of Hodge theory: augmented (anti-)chiral superfield formalism," Annals of Physics, vol. 394, pp. 98-119, 2018.

[22] T. Bhanja, N. Srinivas, and R. P. Malik, "Nilpotent charges of a toy model of Hodge theory and an $N=2$ SUSY quantum mechanical model: (anti-)chiral supervariable approach," International Journal of Modern Physics A: Particles and Fields; Gravitation; Cosmology; Nuclear Physics, vol. 34, no. 30, p. 1950183, 2019.

[23] B. Chauhan, S. Kumar, and R. P. Malik, "Nilpotent charges in an interacting gauge theory and an $N=2$ SUSY quantum mechanical model: (anti-)chiral superfield approach," International Journal of Modern Physics A: Particles and Fields; Gravitation; Cosmology; Nuclear Physics, vol. 34, no. 24, p. 1950131, 2019.

[24] B. Chauhan, S. Kumar, A. Tripathi, and R. P. Malik, "Modified 2D Proca theory: revisited under BRST and (anti-)chiral superfield formalisms," Advances in High Energy Physics, vol. 2020, Article ID 3495168, 38 pages, 2020.

[25] A. Shukla, S. Krishna, and R. P. Malik, "Supersymmetrization of horizontality condition: nilpotent symmetries for a free spinning relativistic particle," European Physical Journal C: Particles and Fields, vol. 72, no. 10, p. 2188, 2012.

[26] B. Chauhan, A. Tripathi, A. K. Rao, and R. P. Malik, "Supervariable and BRST approaches to a toy model of reparameterization invariant theory," https://arxiv.org/abs/1912.12909.

[27] S. Krishna, D. Shukla, and R. P. Malik, "A novel observation in the brst approach to a free spinning relativistic particle," International Journal of Modern Physics A: Particles and Fields; Gravitation; Cosmology; Nuclear Physics, vol. 28, article 1350108, 2018.

[28] A. Shukla, T. Bhanja, and R. P. Malik, "Curci-Ferrari-type condition in Hamiltonian formalism: a free spinning relativistic particle," EPL (Europhysics Letters), vol. 101, no. 5, p. 51003, 2013.

[29] R. P. Malik, "Interacting relativistic particle: time区space noncommutativity and symmetries," International Journal of Modern Physics A, vol. 22, no. 5, pp. 1053-1076, 2012.

[30] R. P. Malik, "Nilpotent symmetries for a spinning relativistic particlein augmented superfield formalism," European Physical Journal C: Particles and Fields, vol. 45, no. 2, pp. 513-524, 2006.

[31] R. P. Malik, "Nilpotent symmetries for a free relativistic particle in augmented superfield formalism," Modern Physics A, vol. 20, no. 23, pp. 1767-1779, 2005.

[32] R. P. Malik, "Noncommutativity in the mechanics of a free massless relativistic particle," Journal of Physics A: Mathematical and Theoretical, vol. 37, no. 50, pp. 12077-12092, 2004.

[33] D. Nemschansky, C. Preitschopf, and M. Weinstein, "A BRST primer," Annals of Physics, vol. 183, no. 2, pp. 226-268, 1988.

[34] M. B. Green, J. H. Schwarz, and E. Witten, Superstring Theory, Vols. 1 and 2, Cambridge University Press, Cambridge, 1987.

[35] J. Polchinski, String Theory, Cambridge University Press, Cambridge, 1998.

[36] P. A. M. Dirac, Lectures on Quantum Mechanics, Belfer Graduate School of Science, Yeshiva University Press, New York, 1964. 
[37] K. Sundermeyer and C. Dynamics, Lecture Notes in Physics, Vol. 169, Springer-Verlag, Berlin, 1982.

[38] M. Faisal and S. Upadhyay, "Spontaneous breaking of the BRST symmetry in the ABJM theory," Physics Letters B, vol. 736, pp. 288-292, 2014.

[39] M. Faisal, S. Upadhyay, and B. P. Mandal, "Finite fielddependent BRST symmetry for ABJM theory in $N=1$ superspace," Physics Letters B, vol. 738, pp. 201-205, 2014.

[40] S. Upadhyay, M. Faisal, and P. A. Ganai, "Interpolating between different gauges in the ABJM theory," International Journal of Modern Physics A: Particles and Fields; Gravitation; Cosmology; Nuclear Physics, vol. 30, no. 27, article 1550185, 2015.

[41] L. Bonora and R. P. Malikin preparation.

[42] S. Weinberg, The Quantum Theory of Fields, Vol. II, Cambridge University Press, Cambridge, 2005. 